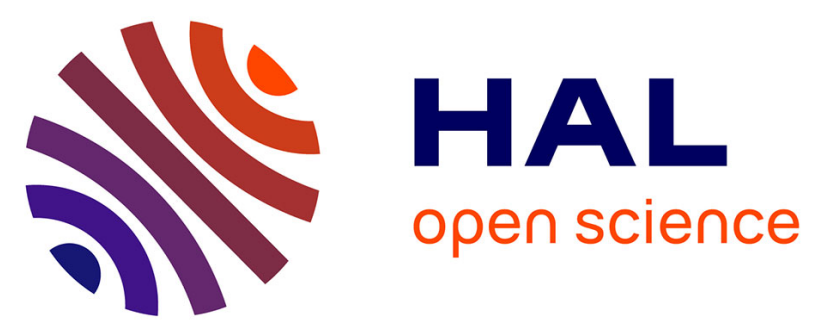

\title{
Linearized Navier-Stokes Equations for Aeroacoustics using Stabilized Finite Elements: Boundary Conditions and Industrial Application to Aft-Fan Noise Propagation
}

Aloïs Bissuel, Grégoire Allaire, Laurent Daumas, Sébastien Barré, Floriane Rey

\section{To cite this version:}

Aloïs Bissuel, Grégoire Allaire, Laurent Daumas, Sébastien Barré, Floriane Rey. Linearized NavierStokes Equations for Aeroacoustics using Stabilized Finite Elements: Boundary Conditions and Industrial Application to Aft-Fan Noise Propagation. Computers and Fluids, inPress. hal-01695874

\section{HAL Id: hal-01695874 \\ https://hal.science/hal-01695874}

Submitted on 29 Jan 2018

HAL is a multi-disciplinary open access archive for the deposit and dissemination of scientific research documents, whether they are published or not. The documents may come from teaching and research institutions in France or abroad, or from public or private research centers.
L'archive ouverte pluridisciplinaire HAL, est destinée au dépôt et à la diffusion de documents scientifiques de niveau recherche, publiés ou non, émanant des établissements d'enseignement et de recherche français ou étrangers, des laboratoires publics ou privés. 


\title{
Linearized Navier-Stokes Equations for Aeroacoustics using Stabilized Finite Elements: Boundary Conditions and Industrial Application to Aft-Fan Noise Propagation
}

\author{
Aloïs Bissuel* Grégoire Allaire ${ }^{\dagger} \quad$ Laurent Daumas* \\ Sébastien Barré* Floriane Rey*
}

Keywords: Aeroacoustics - Finite Elements - SUPG - Boundary conditions - Linearized Navier-Stokes - Linearized Euler

\begin{abstract}
In this paper, a numerical method for solving the linearized Navier-Stokes equations is presented for aeroacoustic sound propagation problem. The Navier-Stokes equations are linearized in the frequency domain. The fan noise of jet engine is emitted nearly selectively at certain frequencies, which depend on the rotation velocity of the fan. A frequency domain approach is highly suitable for this kind of problem, instead of a costly time-dependent simulation which can handle a large range of frequencies depending on the time step and the mesh.

The calculations presented here were all made using Aether, a NavierStokes code which uses finite elements stabilized with SUPG (Streamline Upwind Galerkin). Automatic code differentiation was used to linearize this code. Entropy variables bring interesting mathematical properties to the numerical scheme, but also prevent the easy implementation of boundary conditions. For instance, the pressure is a non-linear combination of the entropy variables. Imposing a pressure variation needs a linearization of this relation which is detailed herein. The performance of different types of boundary conditions used to impose the acoustic pressure variation inside the engine is studied in detail. Finally, a very surprising effect of the SUPG scheme was to transform a homogeneous Dirichlet boundary condition on all variables to a transparent one which is able to let only outgoing waves pass

\footnotetext{
*Dassault Aviation 78, quai Marcel Dassault 92552 Saint-Cloud CEDEX 300 France e-mail: alois.bissuel@dassault-aviation.com

${ }^{\dagger}$ Centre de Mathématiques Appliquées, UMR CNRS 7641 École Polytechnique, Université Paris-Saclay, 91128 Palaiseau, France e-mail: allaire@cmap.polytechnique.fr
} 
through with no incoming wave. A one-dimensional toy model is given to explain how SUPG brings about this transformation.

The last part of the article is dedicated to an industrial test case. The geometry of a model turbine from the Clean Sky European project was used for sound propagation of the fan exhaust noise of a jet engine. Computations on several modes with increasing complexities were done and the results compared to a boundary element method which served as a reference when no mean flow is present. Results of a computation with a mean flow are shown.

\section{Introduction}

Aircraft manufacturers face ever more stringent noise regulations [13]. They need to be able to predict the noise emitted by aircraft, especially during take-off and approach, the only two phases of flight during which the aircraft is close to the ground. The noise emitted by an aircraft can be split into two components. Airframe noise is the first one, and is due to unsteadiness of the flow. Turbulence in the flow and cavities in the surface of the aircraft can all generate unsteadiness, and each gives rise to noise with very different spectral contents [14]. The second noise component comes from the engine. It can itself be split into two main sources, which are the fan noise coming from the rotating parts of the engine, and the jet noise, where very high turbulence generates large pressure fluctuations. The engine noise is predominant especially at take-off, during which the engines run at full power.

In this paper, the propagation of engine fan noise is investigated. In this case, acoustic perturbations stem from rotating parts. They are thus emitted selectively at some frequencies, which depend on the rotation velocity of the engine. A frequency-domain linearization of the Euler or Navier-Stokes equations is the method of choice for such problems where computations can be done for one frequency at a time.

The exhaust fan noise propagation combines different physical phenomena. The sound waves are diffracted and reflected by the engine and aircraft surface, and they are also refracted by the velocity gradients at the interface of the jet. While the two former phenomena can be reduced to integral computations on the surface, the latter can only be computed by volume methods as the base flow is not potential [2].

The finite element method is often used in computational fluid dynamics [3]. Its main advantages lie in the natural handling of unstructured meshes, which enable complex geometries to be easily handled, and strict mathematical setting for convergence proof. The use of linearized Euler equations solved with a finite element method for aeroacoustic is not new [6, 11, 12], though this paper might be the first one in which an industrial use of this technique is presented. 
The industrial test case presented here is a model turbine from the Clean Sky European project. This project aims at developing environmentally friendly aircraft which would burn less fuel and also emit less noise. As part of the Smart Fixed Wing Aircraft, one of the six platforms of the Clean Sky project, Dassault Aviation is working on the integration of aft-body concepts for engine noise shielding [13]. An innovative U-tail aircraft model equipped with turbine powered simulators was wind-tunnel tested to assess the acoustic shielding effect of the U-tail on a typical business jet with side-mounted engines.

In this paper, the Navier-Stokes code Aether which was used for the calculations is first presented, along with all the steps an aeroacoustic computations requires. Although the use of stabilized finite elements for solving the linearized Euler equations is not new (see [6, 11, 12]), solving the linearized Navier-Stokes equations for aeroacoustic noise propagation is not a standard industry practice. It is also the first time results of aeroacoustic computations with the code Aether on industrial test cases are published. Afterwards, the boundary conditions used in these computations are presented, with both mathematical settings and numerical tests. One surprising side-effect of the use of SUPG stabilization is that a standard homogeneous Dirichlet boundary condition transformed itself into a transparent boundary condition. A careful mathematical analysis in $1 \mathrm{D}$ shows that Dirichlet boundary conditions are perfect transparent boundary conditions. Though no such analytic demonstration can be done in 2D and 3D (actually, it is easy to see that a Dirichlet boundary condition cannot be exactly transparent), it turns out that, in numerical practice for real test cases, a Dirichlet boundary condition behaves reasonably well as a transparent boundary condition. This enables us to considerably restrict the extent of the computational domain, in turn resulting in smaller computation time. Finally, results on a model turbine engine are presented to display the industrial use of such methods.

\section{The Navier-Stokes code Aether}

Dassault Aviation uses an in-house developed code called Aether for solving the non-linear Navier-Stokes equations on unstructured meshes using finite element modeling [3]. Several turbulence models are available for stationary computations using RANS ( $k-\varepsilon$, Spalart-Allmaras, etc). For unsteady computations, DES and VMS approaches can be used [19]. The NavierStokes equations have been fully implicited, using the GMRES algorithm to solve the linear problem at each Newton-Raphson iteration. The code has been parallelized using MPI for large scale computations. The main numerical ingredients of the code Aether are detailed below. 


\subsection{Main numerical ingredients}

Aether uses entropy variables for solving the Navier-Stokes equations. These equations can be written in quasi-linear form using the conservative variables $\mathbf{U}=\left(\rho, \rho \mathbf{u}, \rho e_{t}\right)$ with $\rho$ being the density, $\mathbf{u}$ the velocity vector and $e_{t}$ the total energy. Using the Einstein summation notation, they read

$$
\mathbf{U}_{, t}+\mathbf{A}_{i} \mathbf{U}_{, i}=\left(\mathbf{K}_{i j} \mathbf{U}_{, j}\right)_{, i}
$$

Here, $\mathbf{U}_{, i}$ denotes a spatial derivative in the direction $i$, and $\mathbf{U}_{, t}$ is the time derivative of $\mathbf{U}$. The matrix $\mathbf{A}_{i}$ is the Jacobian of the Euler flux $\mathcal{F}^{E u l}$ in the direction $i$, so that $\mathbf{A}_{i} \mathbf{U}_{, i}=\mathcal{F}_{, i}^{E u l}$. Similarly, $\mathbf{K}_{i j}$ is the Jacobian in the direction $j$ of the diffusive flux in the direction $i$. To get better numerical properties, Mallet [8, 10] introduced a change of variables. Using the entropy function $\mathcal{H}=-\rho s$, where $s$ is the entropy, the so-called entropy variables are defined as $\mathbf{V}^{T}=\frac{\partial \mathcal{H}}{\partial \mathbf{U}}$. In three dimensions, defining $h$ as the enthalpy, the entropy variables are thus

$$
\mathbf{V}=\frac{1}{T}\left(\begin{array}{c}
h-T s-\frac{\|\mathbf{u}\|^{2}}{2} \\
u_{1} \\
u_{2} \\
u_{3} \\
-1
\end{array}\right)
$$

Introducing the change of basis matrix $\widetilde{\mathbf{A}}_{0}=\mathbf{U}_{\mathbf{V}}$, the non-linear NavierStokes equations in entropy variables are described by equation (3).

$$
\widetilde{\mathbf{A}}_{0} \mathbf{V}_{, t}+\widetilde{\mathbf{A}}_{i} \mathbf{V}_{, i}=\left(\widetilde{\mathbf{K}}_{i j} \mathbf{V}_{, j}\right)_{, i}
$$

The definition of the Euler flux and diffusive matrices simply follows the change of variables, i.e. $\widetilde{\mathbf{A}}_{i}=\mathbf{A}_{i} \widetilde{\mathbf{A}}_{0}$ and $\widetilde{\mathbf{K}}_{i j}=\mathbf{K}_{\mathbf{i j}} \widetilde{\mathbf{A}}_{0}$. Matrices with a tilde indicate that they operate on entropy variables. These matrices have interesting numerical properties. They are all symmetric, $\widetilde{\mathbf{A}}_{0}$ also being positive definite and the block matrix $\widetilde{\mathbf{K}}=\left[\widetilde{\mathbf{K}}_{i j}\right]$ being positive semi-definite [8].

Aether uses the finite element method to solve the Navier-Stokes equations on an unstructured mesh. Complex geometries can easily be meshed with unstructured meshes composed of simplex [3]. Standard finite element methods are not suitable for convection dominated problem, as they are unstable [9, 10]. They need to be stabilized. The authors of [9, 10] generalized the SUPG stabilization method for multi-dimensional system of equations. The standard (Galerkin) finite element discretization of equation (3) stems from its variational form. It is simply obtained by multiplication with a test 
functional $\mathbf{W}$, and then integrated over the whole domain $\Omega$.

$$
\int_{\Omega} \mathbf{W} \cdot\left(\widetilde{\mathbf{A}}_{0} \mathbf{V}_{, t}+\widetilde{\mathbf{A}}_{i} \mathbf{V}_{, i}-\left(\widetilde{\mathbf{K}}_{i j} \mathbf{V}_{, j}\right)_{, i}\right) d \Omega
$$

As stated before, this Galerkin discretization is not stable when the equations are dominated by advection [10]. The SUPG stabilization consists in modifying the test function. $\mathcal{L}_{a d v}$ is the advective operator of equation (3), i.e. $\mathcal{L}_{a d v}(\mathbf{V})=\widetilde{\mathbf{A}}_{i} \mathbf{V}_{, i}$, the stabilized formulation reads

$$
\int_{\Omega}\left(\mathbf{W}+\boldsymbol{\tau} \mathcal{L}_{a d v}(\mathbf{W})\right) \cdot\left(\widetilde{\mathbf{A}}_{0} \mathbf{V}_{, t}+\widetilde{\mathbf{A}}_{i} \mathbf{V}_{, i}-\left(\widetilde{\mathbf{K}}_{i j} \mathbf{V}_{, j}\right)_{, i}\right) d \Omega
$$

This formulation is consistent. This means that the solution of equation (3), which drives the residual $\widetilde{\mathbf{A}}_{0} \mathbf{V}_{, t}+\widetilde{\mathbf{A}}_{i} \mathbf{V}_{, i}-\left(\widetilde{\mathbf{K}}_{i j} \mathbf{V}_{, j}\right)_{, i}$ to zero, is solution to both the stabilized and the standard Galerkin discretizations. The stabilization matrix $\boldsymbol{\tau}$ is symmetric positive definite. The definition of $\boldsymbol{\tau}$ from Mallet [10] for the Euler equations is

$$
\boldsymbol{\tau}=\widetilde{\mathbf{A}}_{0}^{-1}\left(\left(\frac{\partial \xi_{i}}{\partial x_{j}} \frac{\partial \xi_{i}}{\partial x_{k}}\right) \mathbf{A}_{\mathbf{j}} \mathbf{A}_{\mathbf{k}}\right)^{-\frac{1}{2}}
$$

Here $\xi_{i}$ is the $i$ coordinate in the reference element, and $\frac{\partial \xi}{\partial x}$ is the Jacobian of the transformation from the current element to the reference element. This advective $\boldsymbol{\tau}$ is thus defined element-wise. The stabilization term $\mathbf{W}_{, j}^{T} \widetilde{\mathbf{A}}_{j} \boldsymbol{\tau} \widetilde{\mathbf{A}}_{i} \mathbf{V}_{, i}$ is analogous to a diffusion. Stabilizing the Navier-Stokes equations uses nearly the same SUPG matrix $\boldsymbol{\tau}$ as for the Euler equations. When the physical diffusion is high, the diffusion term due to the SUPG stabilization can be scaled down to keep the numerical scheme from being overly diffusive. The details are in Mallet [9, 10.

The code Aether has been linearized using the automatic differentiation tool Tapenade [7]. It has been used for aerodynamic shape optimization [4] and flutter calculations [5]. Its use for aeroacoustics is described here for the first time. The linear system for solving these equations is built as follows. Starting from the non-linear residual $\mathbf{E}$ which is equation (5), the matrix $\mathbf{A}$ of the linear system $\mathbf{A x}=\mathbf{b}$ is simply the jacobian of the residual with respect to the entropy variables $\mathbf{V}$. For a time harmonic problem, the imaginary part of the matrix is the jacobian of the residual with respect to the time derivative of $\mathbf{V}$. Thus the matrix is

$$
\mathbf{A}=\frac{\partial \mathbf{E}}{\partial \mathbf{V}}+i \omega \frac{\partial \mathbf{E}}{\partial \mathbf{V}_{, t}}
$$

The right-hand side of the linear problem has a less universal definition. For aeroelasticity (and direct optimization problem), it is created by volume 


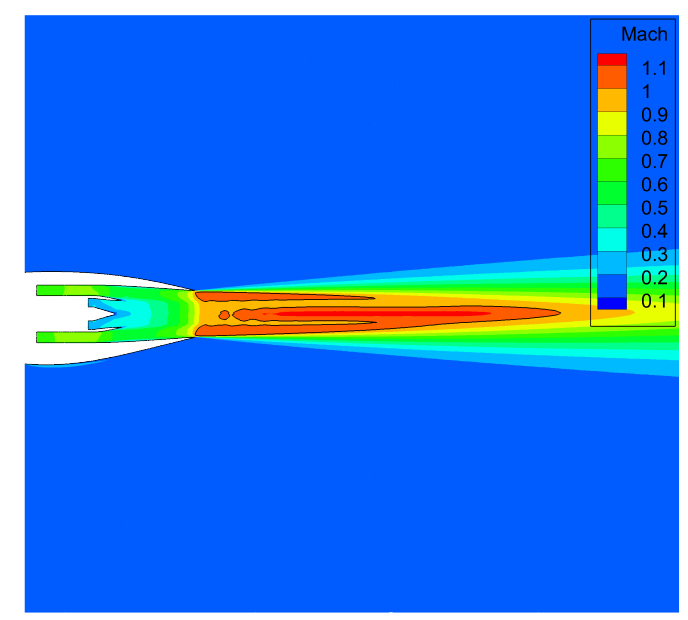

Figure 1: Mach number downstream a jet engine at full-power for take-off

source terms stemming from mesh deformation [5]. In aeroacoustics, the right-hand side comes from non-homogeneous Dirichlet boundary conditions solely which are described below in section 3.1. The matrix $\mathbf{A}$ is sparse as it comes from a finite element discretization method. This non-symmetric linear system is solved using the iterative algorithm GMRES [15]. It minimizes the residual on a basis formed by orthogonalizing successive iterates with the matrix of the starting vector.

\subsection{The steps of an aeroacoustic computation}

An aeroacoustic computation is done in three steps. First, the mean flow has to be computed. Then the linearized Navier-Stokes computation is done. Finally, some sort of post-processing is made to get the quantity of interest, usually the far-field acoustic pressure. In this section, examples are taken from the test case presented in section 4 .

As stated before, the first step is to get a mean flow around which the aeroacoustic computation will be done. Different sorts of computations can be used for this. In figure 1 , a RANS computation was used.

Non-linear Navier-Stokes computations, be they unsteady or time averaged, need a highly detailed mesh near the surface of the object to properly capture the boundary layers. These regions with strong gradients need to be meshed with extremely fine elements in the normal direction to the surface. For the extremely high Reynolds flows typical of external aerodynamic of airplanes, the first element in the boundary layer might be a couple of mi- 


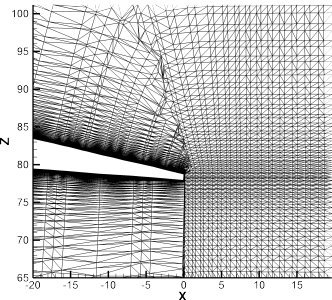

RANS mesh

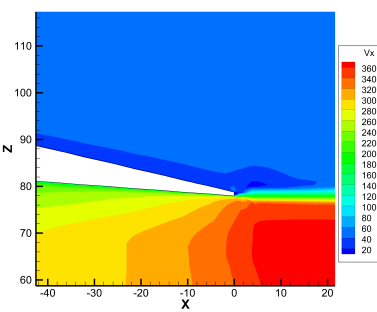

RANS solution

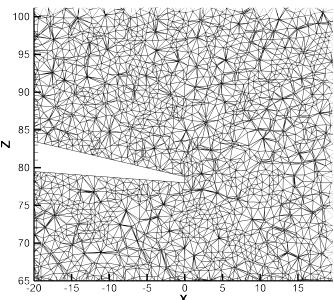

Acoustic mesh

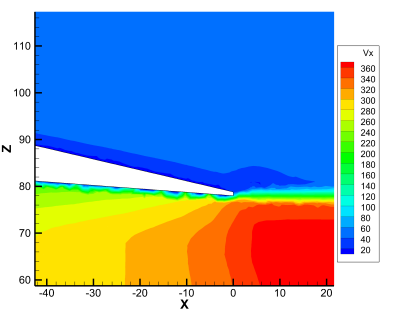

RANS solution on the acoustic mesh

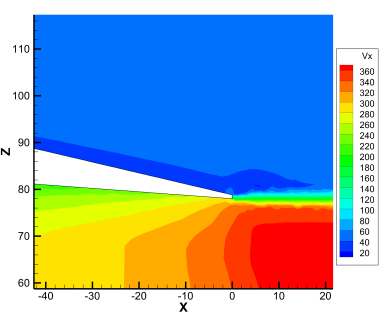

Solution on the acoustic mesh after smoothing

Figure 2: Top: mesh used for the non-linear Navier-Stokes computation and mesh used for sound propagation. Bottom: effect of the projection of the RANS solution onto the acoustic mesh, and smoothing of the boundary layer afterwards

crons high. Because the surface of the object cannot be meshed with such a fine discretization, the elements in the boundary layer are also extremely elongated, with aspect ratios well above the tens of thousands. Figure 2, top left, shows an example of such a mesh.

These meshes cannot be used for aeroacoustic sound propagation, as they are overly fine in the boundary layer region and too coarse elsewhere. The linearized Navier-Stokes computation has to be done on another mesh suited for this task. To be properly captured, sound waves need at least a certain number of nodes per wavelength. The mesh needs also to be quite isotropic, as the sound waves can propagate in all directions. The size of the elements is chosen with respect to the highest intended frequency.

After projecting the solution onto the acoustic mesh, care should be taken to eliminate the remaining boundary layer. First of all, the boundary layer is not resolved by the isotropic elements. This is seen in figure 2, Secondly, the effect of the boundary layer on the acoustic propagation is neglected [14], as being negligible compared to diffraction by the object and refraction by the mean flow. An appropriate volume smoothing was used to remove the boundary layer, as seen in figure 2. The mean flow field on the acoustic mesh after smoothing shows no large difference with the solution on the RANS mesh. 


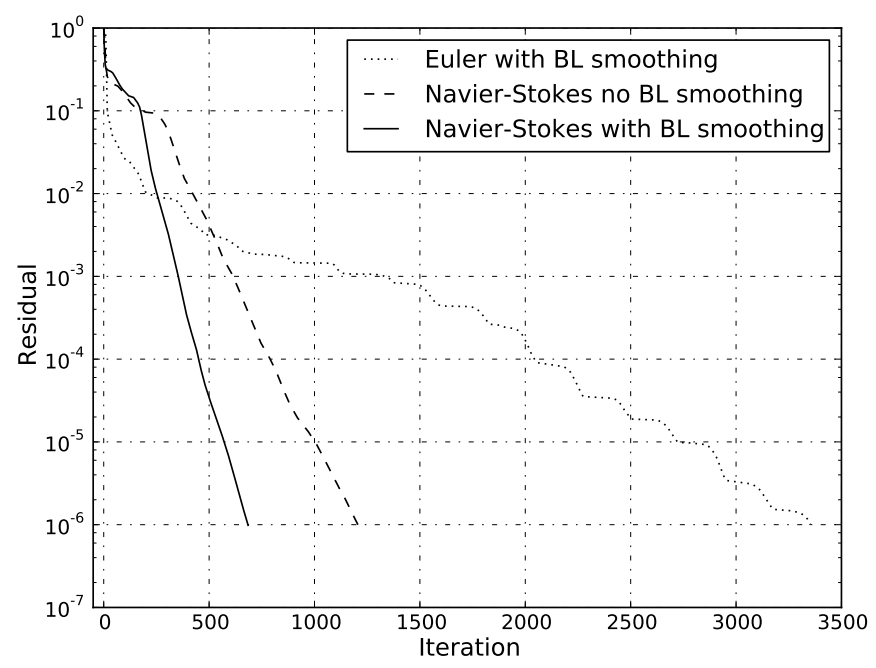

Figure 3: Normalized residual of the GMRES iterations for solving the linear system. Computation of a plane mode at $2 \mathrm{kHz}$ on a coarse mesh comparing linearized Euler and linearized Navier-Stokes, with and without smoothing of the boundary layer. Linearized Euler without boundary layer smoothing failed to converge

Acoustic sound propagation is governed by the Euler equations. Viscosity effects are not of interest in our case. Atmospheric sound attenuation over distance is not taken into consideration. Acoustic boundary layer is neglected for aeroacoustic application. Thus, there are no obvious advantages which can be conceived beforehand to use the linearized Navier-Stokes equations instead of the linearized Euler equations. Some numerical experiments showed us that using linearized Navier-Stokes gives better convergence on some cases. Figure 3 shows the convergence of GMRES residuals, normalized so that the first residual is of norm 1, comparing the convergence of linearized Euler and Navier-Stokes equations on a coarse mesh, with or without smoothing the boundary layer of the mean flow. From this graph, it is clear that the Navier-Stokes equations enable a faster convergence, and a higher tolerance to unresolved gradients, such as the one found in the boundary layer when not smoothed. It is also interesting to note that the linearized Euler equations failed to converge when the boundary layer of the mean flow was not smoothed. This indicates a clear gain of robustness of using the full Navier-Stokes equations. As a last comment, it should be duly noted that the results were completely the same, regardless of the equations used. This was made possible by using the same boundary conditions on the surface of the object, i.e. slip boundary conditions which are the only one available when computing Euler solutions.

On a finer mesh, the overall picture looks quite different, as presented on 


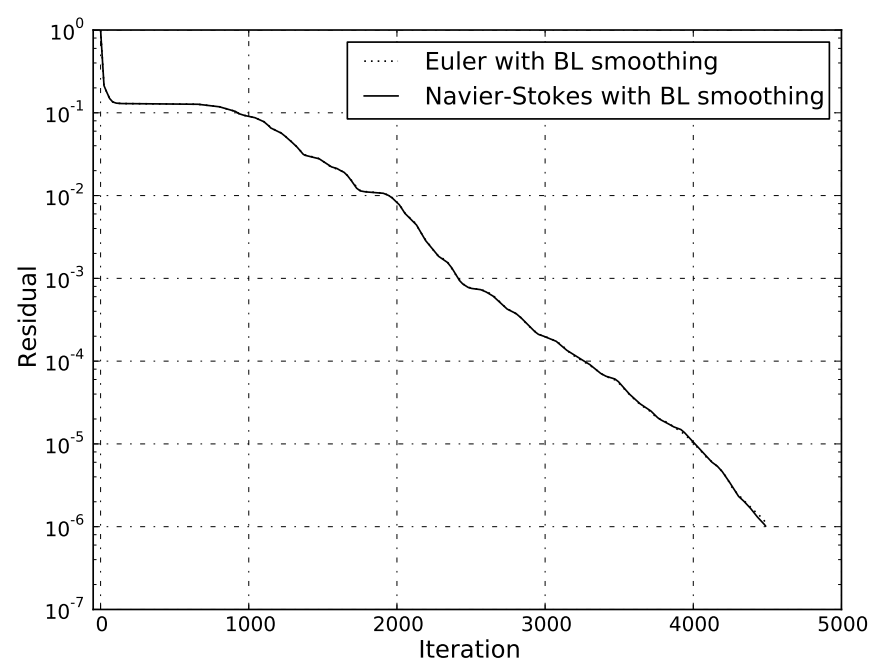

Figure 4: Normalized residual of the GMRES iterations for solving the linear system. Computation of a plane mode at $2 \mathrm{kHz}$ on a high quality mesh comparing linearized Euler and linearized Navier-Stokes with boundary layer smoothing.

figure 4. The convergence curves of the linearized Euler and Navier-Stokes schemes are hardly distinguishable. In this case, the added viscosity terms of linearized Navier-Stokes do not bring much improvement. The added cost of these terms is negligible, as they are only computed to build the matrix, which takes little time compared to the resolution of the linear system. To conclude, it seems that using the Navier-Stokes equations may bring some robustness in cases where the mesh is not perfect, and where some gradients of the mean flow are not perfectly resolved. Thus, linearized Navier-Stokes was always used to be sure of not running into convergence troubles if the smoothing step or the mesh were not good enough.

The computation of the sound propagation gives the near-field solution. The far-field solution is of interest to characterize the sound footprint of an aircraft. As extending the volume mesh to far-field distances would be costprohibitive, another method is used for propagating the near-field solution to great distances. The far field medium is supposed to be homogeneous with a uniform sound velocity and no diffracting object. The propagation of sound waves has an analytic solution using Green function. Using information on an interpolating surface near the aircraft, the sound is propagated to arbitrary distances using the Kirchhoff method. 


\section{Boundary conditions}

Aeroacoustic computations using the code Aether used three types of Dirichlet boundary conditions. As explained in section 2.2, slip boundary conditions, which are typical of Euler equations, were used at the boundary of the object. When computing the propagation of jet engine fan noise, the sound perturbation induced by the fan is first projected onto a modal basis. In cylinders, it is easy to give the exact form of these modes [14]. The computation of the propagation of the whole fan noise is done on its leading modal components separately, which are then recombined. The modes are imposed on a plane called the modal plane, located near the true location of the fan in the real engine. On the modal plane, modes were injected using non-homogeneous Dirichlet boundary condition on the pressure only, or on the incoming characteristic. Finally, the far-field boundary condition was a simple homogeneous Dirichlet one, which proved transparent. These two latter types of conditions are explained below.

\subsection{Inhomogeneous Dirichlet boundary condition}

Recalling section 2.1, the Navier-Stokes code Aether uses entropy variables. The five variables, a chemical potential, velocities divided by temperatures, and minus the inverse of the temperature, are not natural quantities to handle. Following the work of Shakib [17, the next paragraphs will explain how to impose a quantity which depends non-trivially on the entropy variables.

\subsubsection{Imposing a pressure variation}

To impose an acoustic pressure variation $d p$ on the linearized system, a linear relation linking $d p$ and the linearized entropy variables $d V_{i}$ has to be found. This linear formula is simply the linearization of the nonlinear relation linking the pressure $p$ and the entropy variables $V_{i}$. This nonlinear formula is:

$$
p=\exp \left(\frac{1}{R}\left(V_{1}-\frac{V_{2}^{2}+V_{3}^{2}+V_{4}^{2}}{2 V_{5}}+C\right)-\frac{\gamma}{\bar{\gamma}}\left(1+\ln \left(-V_{5}\right)\right)\right)
$$

Here $\gamma$ is the ratio of specific heats i.e. $\gamma=c_{p} / c_{v}$ and $\bar{\gamma}$ represents $\gamma-1$. $C$ is a constant. Note that the pressure depends non linearly on all the variables. As imposing the pressure through variables which only depend on temperature and/or velocities (i.e. $V_{2}, \cdots, V_{5}$ ) would make little sense, the first variable will be used for the Dirichlet condition. Inverting the previous expression gives 


$$
\begin{aligned}
V_{1} & =R \ln p+\frac{V_{2}^{2}+V_{3}^{2}+V_{4}^{2}}{2 V_{5}}-C+\frac{R \gamma}{\bar{\gamma}}\left(1+\ln \left(-V_{5}\right)\right) \\
& =f_{p}\left(V_{2}, V_{3}, V_{4}, V_{5}, p\right)
\end{aligned}
$$

Linearizing the expression gives

$$
d V_{1}=\sum_{i=2}^{5} \frac{\partial f_{p}}{\partial V_{i}} d V_{i}+\frac{\partial f_{p}}{\partial p} d p
$$

The variations of the variable depend linearly on each other, and the coefficients are non-linear functions of the variables where the linearization was made:

$$
\begin{gathered}
\frac{\partial f_{p}}{\partial V_{2}}=\frac{V_{2}}{V_{5}} \quad \frac{\partial f_{p}}{\partial V_{3}}=\frac{V_{3}}{V_{5}} \quad \frac{\partial f_{p}}{\partial V_{4}}=\frac{V_{4}}{V_{5}} \\
\frac{\partial f_{p}}{\partial V_{5}}=-\frac{V_{2}^{2}+V_{3}^{2}+V_{4}^{2}}{2 V_{5}^{2}}+\frac{R \gamma}{\bar{\gamma}} \frac{1}{V_{5}} \quad \frac{\partial f_{p}}{\partial p}=\frac{R}{p}
\end{gathered}
$$

The first variable $d V_{1}$ was chosen to impose the pressure boundary condition. It is replaced with the right-hand side of (11). In vector notation, this gives

$$
\mathbf{d V} \longrightarrow \mathbf{S d V}+\left(\begin{array}{c}
\frac{R d p}{p} \\
0 \\
0 \\
0 \\
0
\end{array}\right)=\mathbf{S d V}+\alpha \mathbf{e}_{\mathbf{1}}
$$

Here $\mathbf{e}_{1}$ is the first vector of the canonical basis of $\mathbb{R}^{5}$ and $\alpha=\frac{R d p}{p}$. Imposing the corresponding homogeneous boundary condition is simply a matter of setting $\alpha$ to zero. The matrix $\mathbf{S}$ is defined as

$$
\mathbf{S}=\left(\begin{array}{ccccc}
0 & \frac{\partial f_{p}}{\partial V_{2}} & \frac{\partial f_{p}}{\partial V_{3}} & \frac{\partial f_{p}}{\partial V_{4}} & \frac{\partial f_{p}}{\partial V_{5}} \\
0 & 1 & & & \\
\vdots & & \ddots & & \\
0 & & & & 1
\end{array}\right)
$$

The matrix $\mathbf{S}$ transforms only the five variables stored at a single node. To impose a boundary condition on a full linear system, it is necessary to index the matrix $\mathbf{S}_{i}$ and the parameter $\alpha_{i}$ by $i$, which is the label of the node. 
To transform the full square matrix of size $5 \times N_{n p}$, the full transformation matrix $\mathbf{S}$ is simply defined by block as

$$
\mathbf{S}=\left(\begin{array}{ccccccc}
\mathbf{I}_{5} & & & & & & \\
& \ddots & & & & & \mathbf{O} \\
& & \mathbf{I}_{5} & & & & \\
& & & \mathbf{S}_{\mathbf{i}} & & & \\
& & & & \mathbf{I}_{5} & & \\
& \mathbf{O} & & & & \ddots & \\
& & & & & & \mathbf{I}_{5}
\end{array}\right)
$$

A similar re-indexing has to be made for the vector $\mathbf{e}_{1}$ which is written $\mathbf{e}_{i, 1}$. The linear system $\mathbf{A x}=\mathbf{b}$, when accounting for the non-homogeneous Dirichlet boundary condition at the node $i$ becomes

$$
\mathbf{A}\left(\mathbf{S} \mathbf{x}+\alpha_{i} \mathbf{e}_{i, 1}\right)=\mathbf{b}
$$

The transformation $\mathbf{M x} \rightarrow \mathbf{M S x}+\alpha \mathbf{e}_{1}$ amounts to combining columns on the matrix M. It can be seen as imposing the inhomogeneous Dirichlet boundary condition on the trial space. The test space should verify the corresponding homogeneous Dirichlet boundary conditions. This amounts to combining lines of the matrix (and of the right-hand side). Thus, the linear system should be left multiplied by $\mathbf{S}^{T}$, which gives

$$
\mathbf{S}^{T} \mathbf{A S x}=\mathbf{S}^{T}\left(\mathbf{b}-\alpha_{i} \mathbf{A} \mathbf{e}_{i, 1}\right)
$$

This procedure modifies the linear system so as to impose the inhomogeneous Dirichlet boundary condition on the node $i$. One can simply iterate this procedure on all the nodes on the border requiring such a condition. An implementation more suited to a finite element setting is to apply such transformation on local matrices when one of its nodes is on the boundary. Thus, the combination of lines and columns can be done on compact elemental matrices, which is much easier than after they are distributed in a global matrix stored in sparse format.

\subsubsection{Imposing incoming acoustic pressure with characteristics}

The previous boundary condition imposes total pressure. This means that the sum of the pressure of the incoming and the outgoing waves is imposed. No control is given on the incoming wave. The outgoing wave, which is reflected back to the modal plane, depends on the geometry of the problem. Comparing two computations with the same imposed total pressure which differ by changes in the geometry can be difficult. The outgoing wave has changed, and thus the energy injected in the system is no longer the same. 
Several techniques exist to get precise control of the injected energy. They all stem from transparent boundary conditions, on which a wealth of literature exists [18. Usually, they are used to mimic the computation of a problem in an unbounded domain in a bounded one. The far-field behavior is modeled through the boundary condition. For acoustics, it implies that only outgoing waves are allowed to pass through the boundary. For the modal plane, one has to let all the outgoing modes and impose only one incoming mode.

One of the easiest techniques for transparent boundary conditions is the use of Euler characteristics. Let us consider that the boundary along which the characteristic boundary condition will be imposed is normal to the $\mathbf{e}_{x}$ direction. Diagonalizing the Euler flux Jacobian computed along this direction gives five eigenvectors (in 3D), which are called the characteristic variables:

$$
\mathbf{d} \mathbf{W}=\left(\begin{array}{c}
d W_{1} \\
d W_{2} \\
d W_{3} \\
d W_{4} \\
d W_{5}
\end{array}\right)=\left(\begin{array}{c}
d \rho-\frac{1}{c^{2}} d p \\
d u_{y} \\
d u_{z} \\
d u_{x}+\frac{1}{\rho c} d p \\
-d u_{x}+\frac{1}{\rho c} d p
\end{array}\right)
$$

Quantities with the prefix $d$ refer to wave quantities and not the the mean flow. The first three characteristic variables correspond to the convection at a speed $u_{x}$ of respectively an entropy wave and two vorticity waves. The characteristic variable $d W_{4}$ represents the convected acoustic wave propagating at a velocity $u_{x}+c$. So is $d W_{5}$, at a velocity $u_{x}-c$. Imposing only the incoming acoustic mode means imposing only the incoming characteristic $d W_{4}$ of velocity $u_{x}+c$. The other characteristics are free. Using the modal theory of sound propagation in tubes [14], one can easily compute the pressure and the acoustic velocity and thus the incoming characteristic $d W_{4}$.

From the definition of $d W_{4}$, imposing the incoming characteristic introduces an affine dependence between the pressure and the normal velocity. One needs to replace the variation of pressure $d p$ in (11) using the definition of $d W_{4}$ in (17).

$$
d p=\rho c d W_{4}-\rho c d u_{x}
$$

From the definition of the entropy variables $\mathbf{V}$ (see section 2.1), $u_{x}=-\frac{V_{2}}{V_{5}}$. Hence $d u_{x}=-\frac{1}{V_{5}} d V_{2}+\frac{V_{2}}{V_{5}^{2}} d V_{5}$. Therefore,

$$
d p=\rho c d W_{4}+\frac{\rho c}{V_{5}} d V_{2}-\rho c \frac{V_{2}}{V_{5}^{2}} d V_{5}
$$

Replacing $d p$ in (11) gives the partial derivatives of the implicit function 
$f_{W_{4}}:$

$$
d V_{1}=\sum_{i=2}^{5} \frac{\partial f_{W_{4}}}{\partial V_{i}} d V_{i}+\frac{\partial f_{W_{4}}}{\partial W_{4}} d W_{4}
$$

Using the definition of the speed of sound for a perfect gas $c^{2}=\gamma R T$ and the state law of the perfect gas $p / \rho=R T$, the partial derivatives of $f_{W_{4}}$ are

$$
\begin{gathered}
\frac{\partial f_{W_{4}}}{\partial V_{2}}=\frac{V_{2}}{V_{5}}-c \quad \frac{\partial f_{W_{4}}}{\partial V_{3}}=\frac{V_{3}}{V_{5}} \quad \frac{\partial f_{W_{4}}}{\partial V_{4}}=\frac{V_{4}}{V_{5}} \\
\frac{\partial f_{W_{4}}}{\partial V_{5}}=-\frac{V_{2}^{2}+V_{3}^{2}+V_{4}^{2}}{2 V_{5}^{2}}+\frac{R \gamma}{\bar{\gamma}} \frac{1}{V_{5}}+c \frac{V_{2}}{V_{5}} \quad \frac{\partial f_{W_{4}}}{\partial W_{4}}=\frac{\gamma R}{c}
\end{gathered}
$$

\subsection{Testing the pressure boundary condition}

A simple tuned cavity was used to test the characteristic boundary condition. A tuned cavity means that the real part of the incoming wave and the outgoing wave are in phase. Their imaginary part cancels each other.

\subsubsection{Test with a plane wave}

For a plane wave, tuning a cavity means choosing a frequency so that the length of the cavity is a multiple of $\lambda / 2$, where $\lambda$ is the wavelength. Using the pressure boundary condition as described in section 3.1.1 means imposing the sum of the incoming and the outgoing waves. Using the pressure boundary condition on the incoming wave only as explained in section 3.1 .2 will make the amplitude of the standing wave two times higher than in the previous case. Figure 5 shows the real part of the standing wave created in such a cavity. For each type of boundary condition, a mode of one Pascal of amplitude was used. As expected, when using characteristics, the amplitude of the standing wave is twice higher than when imposing the total pressure.

\subsubsection{Accuracy with complex modes}

Other modes than a plane wave can propagate in infinite tubes. An infinity of complex modes exists, each with a cut-off frequency below which it cannot propagate. A tuned cavity with these complex modes is considered. It consists in a tube of radius $R_{0}$ with a length $L$. A system of orthoradial coordinates $(r, \theta, x)$ is fitted to the tube. The tube is blocked at $x=L$ with a perfectly reflective boundary. The modes are introduced on the plane $x=0$. For complex modes, the tuning formula to determine all resonance frequencies for a given mode is more complicated than for a plane wave, where it is simply a matter of setting the length of the cavity to a multiple 


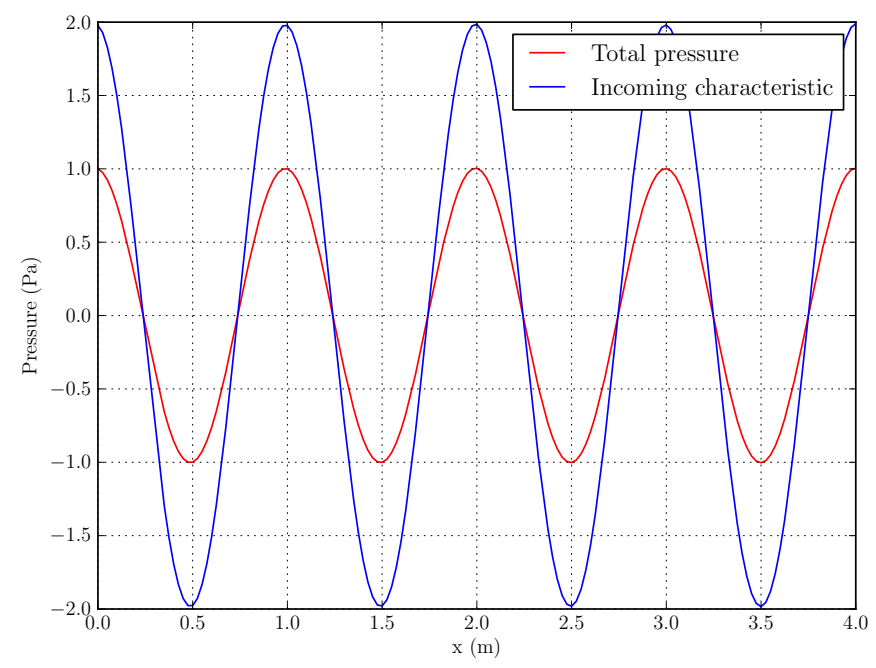

Figure 5: Real part of the pressure variation. Plane mode in a tuned cavity at $340 \mathrm{~Hz}$

of half wavelengths. The general form of these complex rotating modes is given below [14].

$$
P_{m, n}(r, \theta)=J_{m}\left(k_{m, n} r\right) e^{i\left(\omega t-m \theta+k_{x} x\right)}
$$

This is the form of the $(m, n)$ mode. The plane mode is $(m=0, n=1)$. $J_{m}$ is the $\mathrm{m}^{\text {th }}$ order Bessel function of the first kind. The radial wave number $k_{m, n}$ is such that $k_{m, n} R_{0}=\chi_{m, n}$ is the $\mathrm{n}^{\text {th }}$ zero of $J_{m}^{\prime}$ the derivative of the Bessel function. At $r=R_{0}$, the pressure field has a zero radial derivative, so as to verify the boundary condition $\frac{\partial P}{\partial \mathbf{n}}=0$. The radial wave number and the axial wave number follow the dispersion relation

$$
k^{2}=k_{x}^{2}+k_{m, n}^{2}
$$

The total pressure field is the sum of the incoming and outgoing waves. As the cavity is closed on one side, their amplitude is the same as the wave bounces back without losing energy. They differ only by the sign of the axial wave number $k_{x}$ and a difference in phase $\phi$. The total pressure field writes

$$
P_{t o t}=J_{m}\left(k_{m, n} r\right) e^{\mathrm{i} \omega t}\left(e^{\mathrm{i}\left(k_{x} x-m \theta\right)}+e^{\mathrm{i}\left(-k_{x} x-m \theta+\phi\right)}\right)
$$

The tuned cavity has a length $L$. At $x=L$ there is a wall, where the pressure follows $\frac{\partial P}{\partial x}=0$. From there it follows that $\phi=-2 k_{x} L$. The cavity is tuned if the incoming wave is the same as the outgoing wave at $x=0$, i.e. if

$$
1=e^{-2 \mathrm{i} k_{x} L}
$$




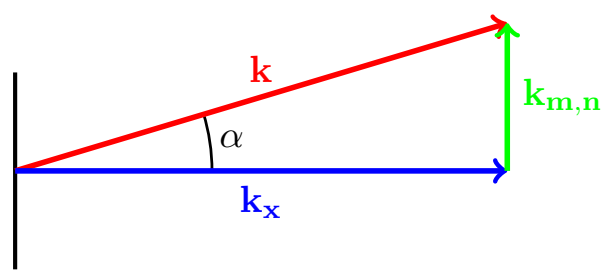

Figure 6: Dispersion relation and definition of the angle $\alpha$

Thus, the axial wave number is entirely defined with the length $L$ of the cavity and $p$, the number of half-wave length fitting in the cavity length.

$$
k_{x}=\frac{p \pi}{L}
$$

Replacing the wave number $k$ by its definition $\frac{2 \pi f}{c}$ in the dispersion relation $(22)$ gives

$$
\left(\frac{2 \pi f}{c}\right)^{2}=\left(\frac{p \pi}{L}\right)^{2}+k_{m, n}^{2}
$$

The frequency as a function of the number of half wavelength in the cavity is thus

$$
f(p)=\sqrt{\left(\frac{p c}{2 L}\right)^{2}+f_{c}^{2}}
$$

$f_{c}=\frac{k_{m, n} c}{2 \pi}$ is the cut-off frequency of the considered mode. For a large $p$, the tuning frequency tends to be the same as for a plane mode, i.e. $f \sim \frac{p c}{2 L}$. The dispersion relation (22) is a pythagorean relation, which enables to define an angle $\alpha$ as shown on the figure 6 .

As $k_{m, n}$ depends only on the mode, the angle $\alpha$ decreases as the frequency grows. At cut-off frequency, $p=0$ so the angle is $90^{\circ}$.

This definition enables the study of the characteristic boundary condition as a function of the angle to the normal of the boundary plane. Transparent boundary conditions using characteristics perform worse when the outgoing wave is not normal to the plane [18. It can be inferred that characteristic boundary conditions used to impose the incoming wave should exhibit the same limitation. Figure 7 shows the loss of pressure depending on the angle $\alpha$ for various modes. Ideally, the ratio between the maximum pressure when imposing total pressure and the maximum pressure when imposing the incoming wave should be two. The delta should be divided by two in order to get closer to the real loss of imposed pressure.

For angles smaller than $45^{\circ}$, the loss of pressure is minimal (less than $10 \%)$. For large angles, it is not clear why the characteristic boundary condition should degrade to the total pressure boundary condition. It is 

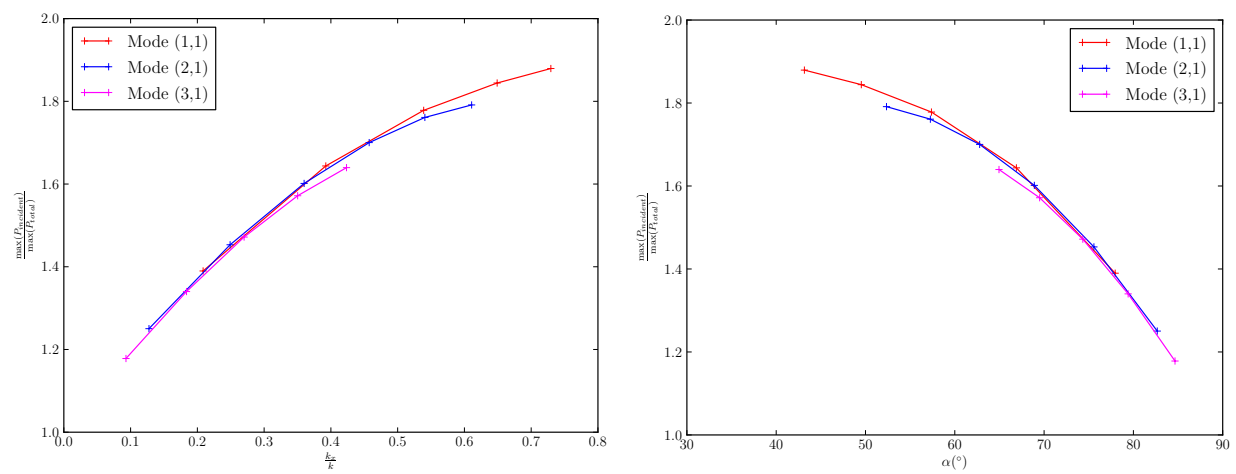

Figure 7: Loss of pressure depending on the angle for various mode as a function of $\frac{k_{x}}{k}$ (left) or as a function of $\alpha$ (right)

quite interesting to see that the behavior of the characteristics does not depend on the mode.

\subsection{Transparent boundary conditions at infinity}

The aeroacoustic calculations using Aether were first made without special consideration regarding the far-field boundary conditions. Quite unexpectedly, simple homogeneous Dirichlet boundary conditions on all five variables turned out to be transparent.

\subsubsection{D explanation of the phenomenon}

To explain this unexpected phenomenon, a 1D model using the linearized Euler equations was used. It enabled the discovery of SUPG stabilization being responsible for turning simple homogeneous boundary condition into transparent one. Recalling section 2.1, the 1D linearized Euler equations can be written using the Jacobian of the Euler flux $\widetilde{\mathbf{A}}_{1}$ as follows

$$
j \omega \widetilde{\mathbf{A}}_{0} \mathbf{V}+\widetilde{\mathbf{A}}_{1} \mathbf{V}_{, x}=\mathbf{0}
$$

$\mathbf{V}$ is the vector of entropy variables for the Euler equations. For the moment, only the convective part will be considered. Adding the complex part, which depends on the frequencys will be done later on. Left-multiplying by the test function $\mathbf{Y}$, integrating and adding the SUPG stabilization term gives

$$
\int_{\Omega^{e}} \mathbf{Y}^{H} \widetilde{\mathbf{A}}_{1} \mathbf{V}_{, x}+\mathbf{Y}_{, x}^{H} \widetilde{\mathbf{A}}_{1} \boldsymbol{\tau} \widetilde{\mathbf{A}}_{1} \mathbf{V}_{, x} d \Omega^{e}
$$

The stabilization matrix $\boldsymbol{\tau}$ is defined as in [9]. For this 1D example, it 
simplifies to

$$
\boldsymbol{\tau}=\widetilde{\mathbf{A}}_{0}^{-1}\left(\left(\frac{\partial \xi}{\partial x} \mathbf{A}_{\mathbf{1}}\right)^{2}\right)^{-\frac{1}{2}}
$$

The term $\frac{\partial \xi}{\partial x}$ is the jacobian of the transformation from the current element to the reference element. From the theory of generalized eigenvalues, there exists two vector basis $\mathbf{S}$ and $\widetilde{\mathbf{S}}$ such that

$$
\begin{aligned}
\left(\mathbf{A}_{\mathbf{1}}-\lambda_{i} \mathbf{I}\right) \mathbf{S}_{i} & =\mathbf{0} \\
\left(\widetilde{\mathbf{A}}_{1}-\lambda_{i} \widetilde{\mathbf{A}}_{0}\right) \widetilde{\mathbf{S}}_{i} & =\mathbf{0} \\
\widetilde{\mathbf{S}}^{T} \widetilde{\mathbf{A}}_{0} \widetilde{\mathbf{S}} & =\mathbf{I}
\end{aligned}
$$

The vectors $\mathbf{S}_{i}$ and $\widetilde{\mathbf{S}}_{i}$ are the column of the matrices $\mathbf{S}$ and $\widetilde{\mathbf{S}}$. These two matrices are also linked by the relation $\mathbf{S}=\widetilde{\mathbf{A}}_{0} \widetilde{\mathbf{S}}$. The normalization relation (32) comes from the fact that $\widetilde{\mathbf{A}}_{1}$ and $\widetilde{\mathbf{A}}_{0}$ are symmetric real matrices (no such relation holds for $\mathbf{S}$ since $\mathbf{A}_{1}$ is not symmetric). From this definition it follows that

$$
\widetilde{\mathbf{S}}^{T} \widetilde{\mathbf{A}}_{1} \widetilde{\mathbf{S}}=\mathbf{S}^{-1} \mathbf{A}_{1} \mathbf{S}=\boldsymbol{\Lambda}
$$

Note that $\mathbf{S}$ diagonalizes the Euler flux Jacobian matrix $\widetilde{\mathbf{A}}_{1}$, so it represents the change of basis matrix from the conservative variables to the characteristic variables. The eigenvalues $\lambda_{i}$ are thus

$$
\boldsymbol{\Lambda}=\operatorname{diag}\left(\lambda_{i}\right)=\operatorname{diag}(u, u+c, u-c)
$$

Using $\widetilde{\mathbf{S}}$ as a change of variables, and introducing the modified coordinates in the trial and test spaces $\mathbf{W}=\widetilde{\mathbf{S}}^{-1} \mathbf{V}$ and $\mathbf{Z}=\widetilde{\mathbf{S}}^{-1} \mathbf{Y}$, the equation reads

$$
\int_{\Omega^{e}} \mathbf{Z}^{H} \widetilde{\mathbf{S}}^{T} \widetilde{\mathbf{A}}_{1} \widetilde{\mathbf{S}} \mathbf{W}_{, x}+\mathbf{Z}_{, x}^{H} \widetilde{\mathbf{S}}^{T} \widetilde{\mathbf{A}}_{1} \boldsymbol{\tau} \widetilde{\mathbf{A}}_{1} \widetilde{\mathbf{S}} \mathbf{W}_{, x} d \Omega^{e}=\mathbf{0}
$$

Using the definition and properties of $\widetilde{\mathbf{S}}$, the first term of the integral in (35) is easily simplified to $\widetilde{\mathbf{S}}^{T} \widetilde{\mathbf{A}}_{1} \widetilde{\mathbf{S}}=\boldsymbol{\Lambda}$. Only the simplification of the second term is a little more involved:

$$
\begin{aligned}
\widetilde{\mathbf{S}}^{T} \widetilde{\mathbf{A}}_{1} \boldsymbol{\tau} \widetilde{\mathbf{A}}_{1} \widetilde{\mathbf{S}} & =\boldsymbol{\Lambda} \widetilde{\mathbf{S}}^{-1} \boldsymbol{\tau} \widetilde{\mathbf{A}}_{0} \widetilde{\mathbf{S}} \boldsymbol{\Lambda} \\
& =\boldsymbol{\Lambda} \widetilde{\mathbf{S}}^{-1} \boldsymbol{\tau} \mathbf{S} \boldsymbol{\Lambda}
\end{aligned}
$$

As $\mathbf{S}$ is the eigenvector basis of $\mathbf{A}_{\mathbf{1}}$, the matrix $\boldsymbol{\tau}$ can be written more simply

$$
\boldsymbol{\tau}=\frac{\partial x}{\partial \xi} \widetilde{\mathbf{A}}_{0}^{-1} \mathbf{S}|\boldsymbol{\Lambda}|^{-1} \mathbf{S}^{-1}
$$


$|\boldsymbol{\Lambda}|$ is the absolute value of the diagonal matrix. Coming back to the simplification of the second term,

$$
\begin{aligned}
\widetilde{\mathbf{S}}^{T} \widetilde{\mathbf{A}}_{1} \boldsymbol{\tau} \widetilde{\mathbf{A}}_{1} \widetilde{\mathbf{S}} & =\boldsymbol{\Lambda} \widetilde{\mathbf{S}}^{-1} \boldsymbol{\tau} \mathbf{S} \boldsymbol{\Lambda} \\
& =\frac{\partial x}{\partial \xi} \boldsymbol{\Lambda} \widetilde{\mathbf{S}}^{-1} \widetilde{\mathbf{A}}_{0}^{-1} \mathbf{S}|\boldsymbol{\Lambda}|^{-1} \mathbf{S}^{-1} \mathbf{S} \boldsymbol{\Lambda} \\
& =\frac{\partial x}{\partial \xi} \boldsymbol{\Lambda} \widetilde{\mathbf{S}}^{-1} \widetilde{\mathbf{S}}|\boldsymbol{\Lambda}|^{-1} \boldsymbol{\Lambda} \\
& =\frac{\partial x}{\partial \xi} \boldsymbol{\Lambda}|\boldsymbol{\Lambda}|^{-1} \boldsymbol{\Lambda} \\
& =\frac{\partial x}{\partial \xi}|\boldsymbol{\Lambda}|
\end{aligned}
$$

The full equation (35) is then diagonal:

$$
\int_{\Omega^{e}} \mathbf{Z}^{H} \boldsymbol{\Lambda} \mathbf{W}_{, x}+\mathbf{Z}_{, x}^{H} \frac{\partial x}{\partial \xi}|\boldsymbol{\Lambda}| \mathbf{W}_{, x} d \Omega^{e}=\mathbf{0}
$$

Using linear function on the element, one can check that this formulation provides a perfect upwinding of the convection term per characteristics. For the present time only the convective term and its stabilization will be considered. The frequency term will be treated afterward. For instance, if $0 \leq u<c$ so that $u-c$ is the only negative eigenvalue, the discretized equations on a uniform mesh of element size $h$ is after division by the volume of the element:

$$
\begin{aligned}
u \frac{w_{i}^{(1)}-w_{i-1}^{(1)}}{h} & =0 \\
(u+c) \frac{w_{i}^{(2)}-w_{i-1}^{(2)}}{h} & =0 \\
(u-c) \frac{w_{i+1}^{(3)}-w_{i}^{(3)}}{h} & =0
\end{aligned}
$$

Perfect upwinding of the convection is achieved and only the third characteristic $w^{(3)}$ has a different discretization of its convective term.

Adding a homogeneous Dirichlet boundary condition on one end node means that all the characteristics there are zero. If this boundary condition is located at the upper-end of the $1 \mathrm{D}$ segment, i.e. at the last discretized node $N$, the first two characteristics will not "see" the boundary condition until they reach it, whereas the third characteristic will be set to zero. This proves that the two first characteristics reach the wall without being affected by it and that the reflected wave (third characteristic) is zero everywhere in the computational domain. This explanation is illustrated on figure 8 . 


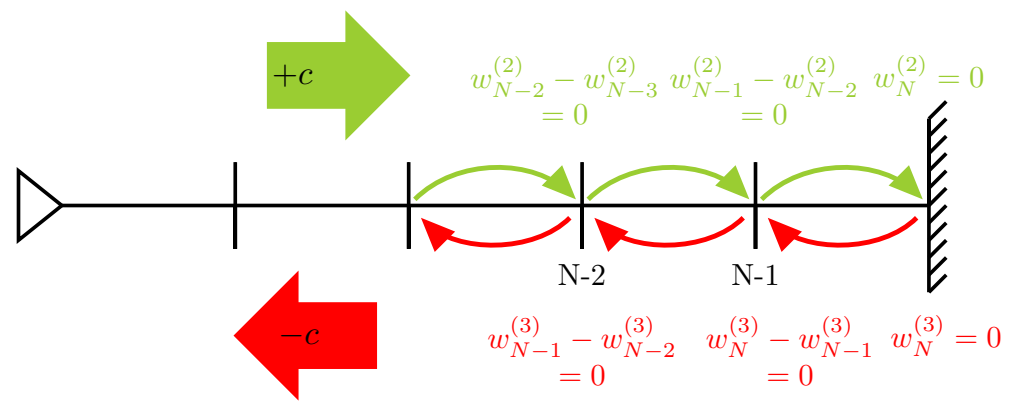

Figure 8: Different upwinding of the two characteristics $w^{(2)}$ and $w^{(3)}$ when using SUPG discretization

\subsubsection{D and $3 \mathrm{D}$ extension}

The SUPG stabilization is proven to give a perfect upwinding for a scalar equation in one dimension [10]. For a symmetric system of variable in one dimension, the stabilization matrix $\boldsymbol{\tau}$ given by Mallet [8] gives the same property. For multidimensional problems, this is unfortunately no longer the case. The stabilization matrix is defined as in Shakib [17]:

$$
\boldsymbol{\tau}=\widetilde{\mathbf{A}}_{0}^{-1}\left(\frac{\partial \xi_{k}}{\partial x_{i}} \frac{\partial \xi_{k}}{\partial x_{j}} \mathbf{A}_{\mathbf{i}} \mathbf{A}_{\mathbf{j}}\right)^{-\frac{1}{2}}
$$

As before, $\frac{\partial \xi}{\partial x}$ is the Jacobian of the transformation from the current element to the reference element. In two (or three) dimensions, there is no basis which diagonalizes simultaneously all flux operators $\mathbf{A}_{\mathbf{i}}$. Thus, it will not be possible to put the system of stabilized equations in a diagonal form as before. Therefore, the stabilization can not be perfect in all directions. Another key point of the stabilization matrix $\tau$ is the definition of the element length. In one dimension, the stabilization term is akin to a diffusive term multiplied by a length, which is the element size. This gives rise to cancellations with some terms of the discretization of the advective flux, and in turn enables a perfect upwinding of the scheme. In three dimensions, the definition of the element size is no longer clear, and does not enable perfect cancellations.

Though the stabilization may not be exact, and the demonstration of the transparency of a simple homogeneous Dirichlet boundary condition no longer feasible, it is of interest to characterize the imperfection of this transparent boundary condition. A simple numerical experiment was set up for it. It is based on the sound propagation in two dimensions from a point monopolar source. As the acoustic power is conserved on every circle whose center is on the source, the acoustic intensity $e_{a c c}$ is proportional to $1 / r$, where $r$ is the distance to the source. The acoustic intensity is proportional to the square of the acoustic pressure, so $p \propto 1 / \sqrt{r}$. 


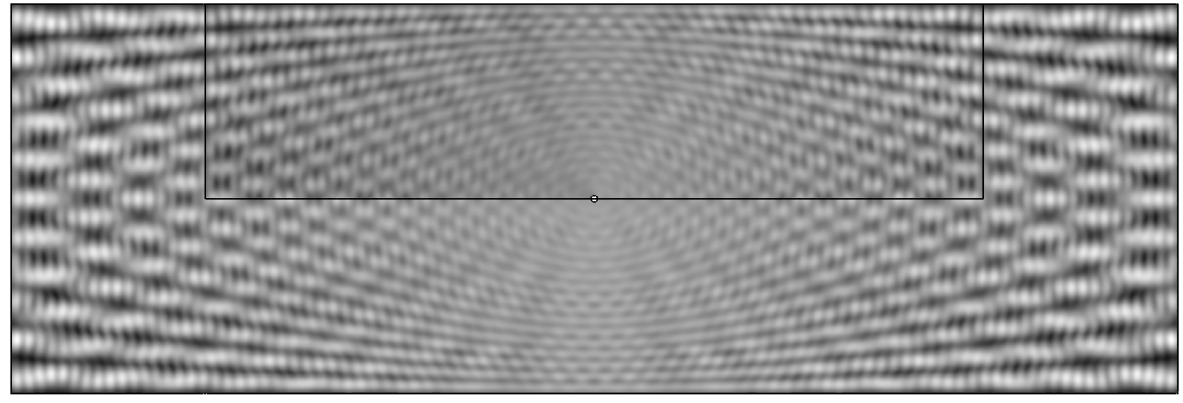

Figure 9: Superposition of the two rectangular computational domains with an acoustic monopole in the center and transparent boundary condition on its edges. The depicted field is $\sqrt{r}|p|$ which is the pressure corrected for $2 \mathrm{D}$ geometric attenuation. The smaller domain is depicted on top of the bigger rectangle.

The results are shown on figure 9 on which the pressure corrected for geometric attenuation is plotted. The first computation was made on a small rectangle with a hole on it exact center where the circular wave is injected. On the edge of this rectangle, homogeneous Dirichlet boundary conditions acting as transparent boundary conditions were used. Only the top half of this first computational domain is shown on the figure. Interference fringes are obvious. They show that some energy is indeed reflected back into the computational domain.

The computation on this first domain was to get a reference, from which the relative difference when modifying the mesh could be computed. Then the mesh was augmented on its left and right sides with symmetric extensions. The bigger domain is depicted underneath the top-half of the first domain. As a first remark, the interference pattern looks absolutely identical to the one in the smaller rectangle. This means that the left and right vertical boundaries are not the boundaries which reflect most of the energy inside the domain. It also demonstrates that the corners do not reflect much energy. This is important to note as corners might be difficult to handle when implementing characteristic boundary conditions. In corners, it is impossible to properly define a normal to the boundary.

Finally, as the distance to the source increases, the interference grows stronger, especially near the horizontal boundaries. This clearly indicates that the interference pattern is due to waves reflected on the horizontal boundaries. The growing amplitude of the interference with the horizontal distance indicates that as the angle to the normal of the boundary increases, the waves are more and more reflected. This is expected for characteristic boundary conditions [18]. 


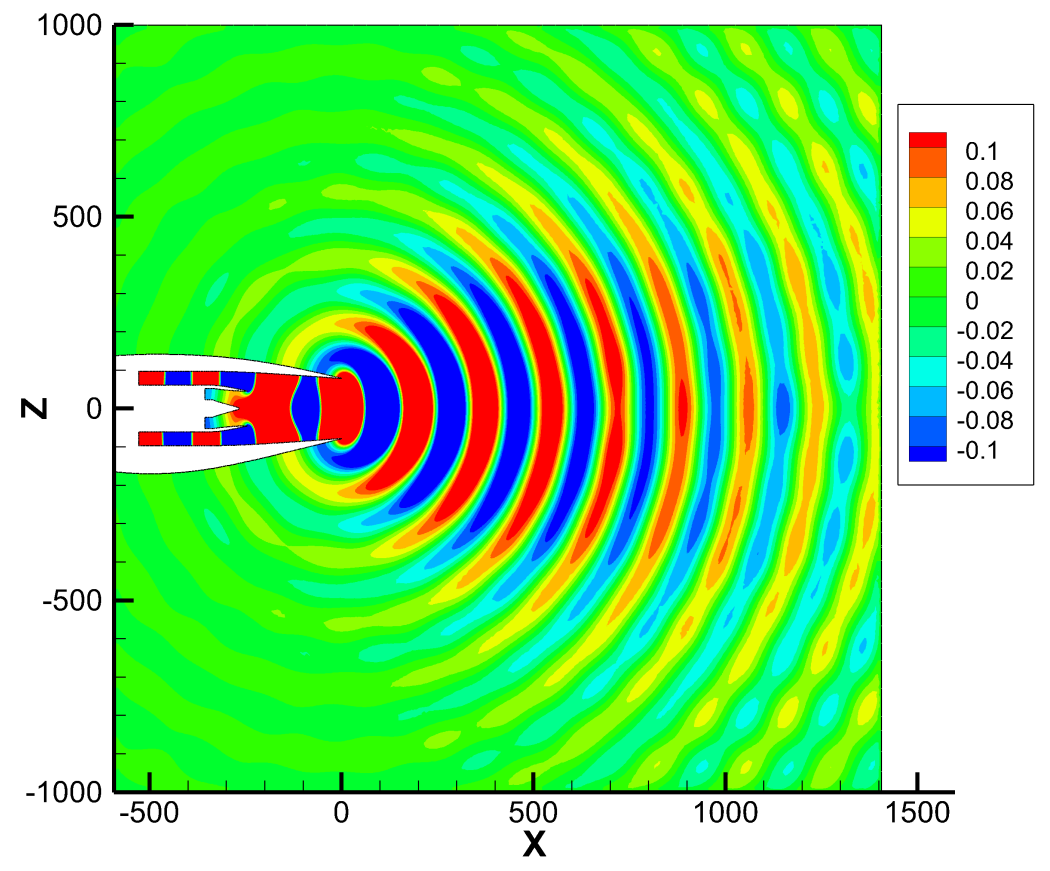

Figure 10: Real part of the pressure variation in Pascal for a plane mode at $2 \mathrm{kHz}$ with no mean flow on a slice of the three dimensional computational domain.

For three dimensional computations, no numerical experiments to characterize precisely the angular behavior of such transparent boundary conditions were done. Only full industrial configurations were tested. For a plane mode at $2 \mathrm{kHz}$ with no mean flow on the jet engine presented afterwards in section 4. the real part of the pressure variation is shown on figure 10. This figure shows the real extent of the three dimensional computational domain on a slice. The far-field boundaries which used transparent boundary conditions were quite close to the exhaust of the engine. Some interference can be seen, but overall it does not radically change the near field solution. Thus these characteristic boundary conditions proved satisfactory as they enabled to use quite small computational domains without altering too much the results.

\subsubsection{A note on the full SUPG coupling}

In section 3.3.1, the linearized Euler equations were considered so that the explanation is clear and simple to grasp. Now, the effect of adding the frequency term in the discretization will be analyzed, with regards to the transparent boundary conditions and to the stability and accuracy of the 
scheme. From the previous section, the standard Galerkin discretization of the linearized Euler equations, written in the characteristic variables, reads

$$
\int_{\Omega^{e}} \mathbf{Z}^{H} j \omega \mathbf{I} \mathbf{W}+\mathbf{Z}^{H} \boldsymbol{\Lambda} \mathbf{W}_{, x} d \Omega^{e}
$$

Their discretization on linear finite elements of uniform length $h$ is given below, where $w_{i+1}^{(2)}$ is the value of the second characteristic at the node $i+1$. A division by $h$ was done so that the spatial derivatives stand out more clearly.

$$
\left\{\begin{array}{l}
j \omega\left(\frac{1}{6} w_{i-1}^{(1)}+\frac{2}{3} w_{i}^{(1)}+\frac{1}{6} w_{i+1}^{(1)}\right)+u \frac{w_{i+1}^{(1)}-w_{i-1}^{(1)}}{2 h}=0 \\
j \omega\left(\frac{1}{6} w_{i-1}^{(2)}+\frac{2}{3} w_{i}^{(2)}+\frac{1}{6} w_{i+1}^{(2)}\right)+(u+c) \frac{w_{i+1}^{(2)}-w_{i-1}^{(2)}}{2 h}=0 \\
j \omega\left(\frac{1}{6} w_{i-1}^{(3)}+\frac{2}{3} w_{i}^{(3)}+\frac{1}{6} w_{i+1}^{(3)}\right)+(u-c) \frac{w_{i+1}^{(3)}-w_{i-1}^{(3)}}{2 h}=0
\end{array}\right.
$$

The convective velocity $u$ is positive and subsonic, so that $u-c$ is the only negative eigenvalue of the Euler flux matrix. The equations stabilized with SUPG, written in terms of entropy variables, read:

$$
\int_{\Omega^{e}}\left(\mathbf{Y}+\boldsymbol{\tau} \widetilde{\mathbf{A}}_{1} \mathbf{Y}_{, x}\right)^{H} \cdot\left(j \omega \widetilde{\mathbf{A}}_{0} \mathbf{V}+\widetilde{\mathbf{A}}_{1} \mathbf{V}_{, x}\right) d \Omega^{e}=\mathbf{0}
$$

Compared to the standard Galerkin method, the test function are modified with the term $\tau \widetilde{\mathbf{A}}_{1} \mathbf{Y}_{, x}$. Two terms are thus added to the equations. They are simply the effect of this change of test function on the frequency and the Euler flux terms. In the characteristic variables, it was already shown that the latter simplifies to $\frac{\partial x}{\partial \xi}|\boldsymbol{\Lambda}|$. The former can also be expressed similarly with the characteristic variables.

$$
\begin{aligned}
\widetilde{\mathbf{S}}^{T} \widetilde{\mathbf{A}}_{1} \boldsymbol{\tau} j \omega \widetilde{\mathbf{A}}_{0} \widetilde{\mathbf{S}} & =\boldsymbol{\Lambda} \widetilde{\mathbf{S}}^{-1} \boldsymbol{\tau} j \omega \mathbf{S} \\
& =j \omega \frac{\partial x}{\partial \xi} \boldsymbol{\Lambda}|\boldsymbol{\Lambda}|^{-1} \\
& =j \omega \frac{\partial x}{\partial \xi} \operatorname{sgn}(\boldsymbol{\Lambda})
\end{aligned}
$$

Here, $\operatorname{sgn}(\boldsymbol{\Lambda})=\operatorname{diag}\left(\operatorname{sgn}\left(\lambda_{i}\right)\right)$, where the function $\operatorname{sgn}(x)$ is 1 if $x \geq 0$ and -1 otherwise. As before, the stabilized equations with the characteristic variables are diagonal and they are given below.

$$
\int_{\Omega^{e}} \mathbf{Z}^{H} j \omega \mathbf{W}+\mathbf{Z}_{, x}^{H} j \omega \frac{\partial x}{\partial \xi} \operatorname{sgn}(\boldsymbol{\Lambda}) \mathbf{W}+\mathbf{Z}^{H} \boldsymbol{\Lambda} \mathbf{W}_{, x}+\mathbf{Z}_{, x}^{H} \frac{\partial x}{\partial \xi}|\mathbf{\Lambda}| \mathbf{W}_{, x} d \Omega^{e}=\mathbf{0}
$$


Their discretization on the same linear finite elements of uniform size $h$ gives

$$
\left\{\begin{array}{l}
j \omega\left(\frac{5}{12} w_{i-1}^{(1)}+\frac{2}{3} w_{i}^{(1)}-\frac{1}{12} w_{i+1}^{(1)}\right)+u \frac{w_{i}^{(1)}-w_{i-1}^{(1)}}{h}=0 \\
j \omega\left(\frac{5}{12} w_{i-1}^{(2)}+\frac{2}{3} w_{i}^{(2)}-\frac{1}{12} w_{i+1}^{(2)}\right)+(u+c) \frac{w_{i}^{(2)}-w_{i-1}^{(2)}}{h}=0 \\
j \omega\left(-\frac{1}{12} w_{i-1}^{(3)}+\frac{2}{3} w_{i}^{(3)}+\frac{5}{12} w_{i+1}^{(3)}\right)+(u-c) \frac{w_{i+1}^{(3)}-w_{i}^{(3)}}{h}=0
\end{array}\right.
$$

Here, the two stabilization terms have each brought in upwinding. The convective term is perfectly upwinded, but the frequency term is also no longer symmetric.

From both discretizations 40) and (43), the recurrence relation of the second characteristic variable $w^{(2)}$ is studied. A solution of the form $w_{n}^{(2)}=$ $\alpha^{n} w_{0}^{(2)}$ is assumed. The parameter $\alpha$ is the solution of a quadratic equation given by the recurrence relation. For instance, for the unstabilized equations (40), the exponent 2 notation being dropped for clarity reasons, this equation is

$$
w_{i-1}\left(\frac{j \omega}{6}-\frac{u+c}{2 h}\right)+w_{i} \frac{2 j \omega}{3}+w_{i+1}\left(\frac{j \omega}{6}+\frac{u+c}{2 h}\right)=0
$$

Introducing $\zeta=\frac{u+c}{\omega h}=\frac{\lambda}{2 \pi h}$, where $\lambda$ is the wavelength, the equation can be further simplified to

$$
w_{i-1}\left(\frac{1}{4}+\frac{3}{4} j \zeta\right)+w_{i}+w_{i+1}\left(\frac{1}{4}-\frac{3}{4} j \zeta\right)=0
$$

The quadratic equation to solve is simply

$$
\left(\frac{1}{4}+\frac{3}{4} j \zeta\right)+X+X^{2}\left(\frac{1}{4}-\frac{3}{4} j \zeta\right)=0
$$

Its solutions are

$$
\alpha_{\text {Gal }}=\frac{-2 \pm \sqrt{3-9 \zeta^{2}}}{1-3 j \zeta}
$$

It is easy to show that for $\zeta>1 / \sqrt{3}$, both roots have a modulus of one. Moreover, $\alpha_{\text {Gal }} \rightarrow-1$ and $\alpha_{\text {Gal }-} \rightarrow 1$ as $\zeta \rightarrow \infty$. The general solution of the recurrence relation (44) is a linear combination of the two solutions which were found, i.e. $w_{i}=\alpha_{G a l-}^{i} w_{0}^{-}+\alpha_{G a l+}^{i} w_{0}^{+}$. The modulus of both $\alpha_{\text {Gal }}$ is equal to one, so the scheme is stable. But the real part of $\alpha_{\text {Gal+ }}$ is negative, so $\alpha_{G a l+}^{i} w_{0}^{+}$will oscillate from node to node. This is unphysical, as the period of the oscillation is fixed by the mesh size and will always exist even if $h \rightarrow 0$. 


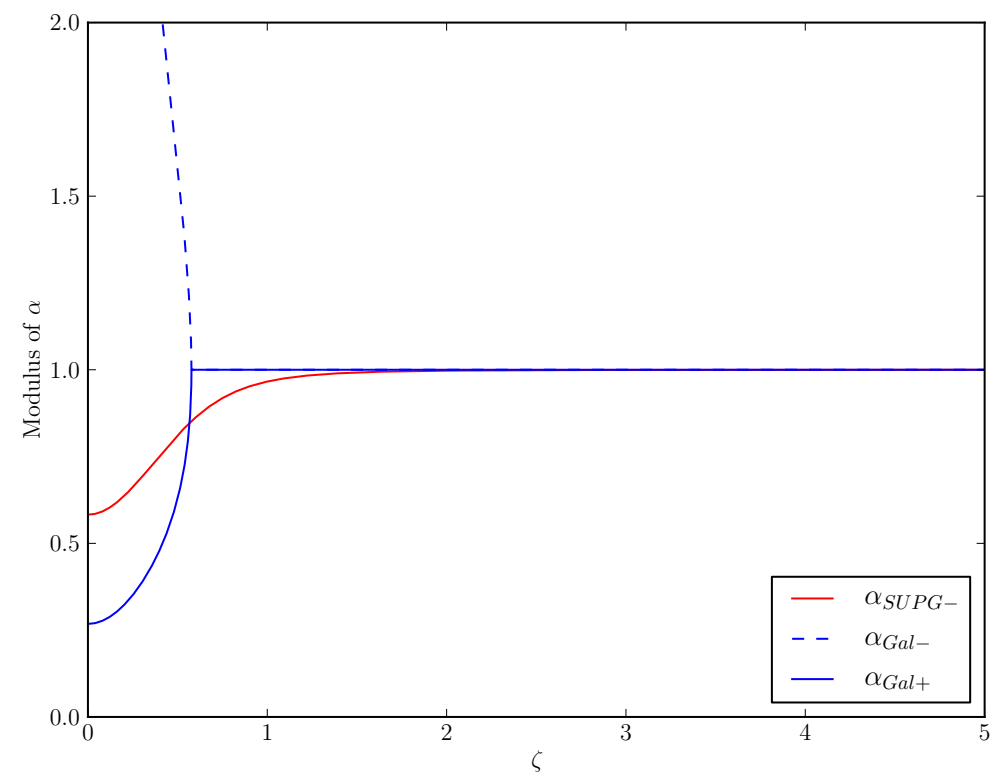

Figure 11: Modulus of the coefficients $\alpha$ introduced in (47) and 49)

For the stabilized equation (43), the resulting quadratic equation is

$$
(-5-12 j \zeta)+(-8+12 j \zeta) X+X^{2}=0
$$

The two roots of this equation are the following

$$
\alpha_{S U P G \pm}=4-6 j \zeta \pm \sqrt{21-36 j \zeta-36 \zeta^{2}}
$$

No simple analytic formula was found for the modulus of both of these roots. They also have each a different limit which is

$$
\begin{array}{r}
\alpha_{S U P G+} \longrightarrow \infty \\
\alpha_{S U P G-} \longrightarrow 1
\end{array}
$$

The norm of the recurrence coefficients $\alpha_{ \pm}$is shown on figure 11 for the Galerkin and SUPG discretizations. Figures 12 and 13 show respectively the real part of the various $\alpha$ and their path on the complex plane. Only the modulus $\alpha_{S U P G+}$ is not shown on the figures as for $\zeta=0,\left|\alpha_{S U P G+}\right| \approx 8.5$ and its modulus grows with $\zeta$ and at infinity, its equivalent is $\left|\alpha_{S U P G+}\right| \sim 12 \zeta$. For all purposes, $\zeta$ is always greater than 1 , as $\zeta=1$ corresponds to roughly 6 nodes per wavelength. Thus, the real part of $\alpha_{S U P G-}$ is positive, as for $\alpha_{\text {Gal-. }}$. The real part of $\alpha_{G a l+}$ is negative. Also, $\left|\alpha_{S U P G-}\right|<1$, as it can be seen graphically and proved for large $\zeta$ by the equivalent described in equation (53). 


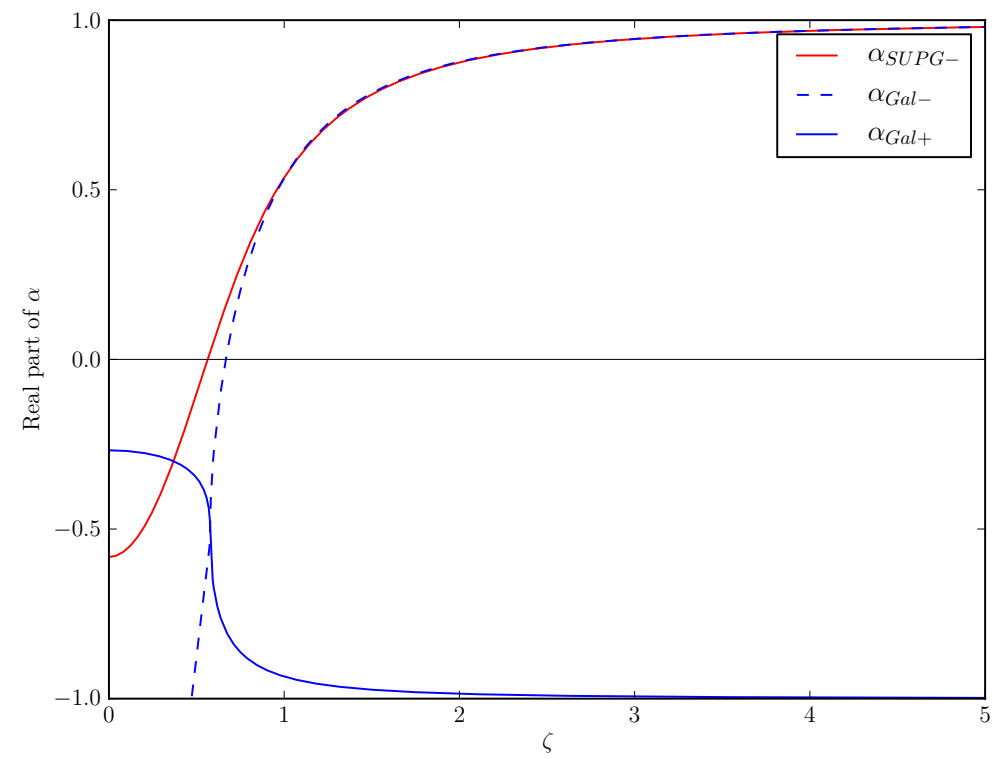

Figure 12: Real part of the coefficients $\alpha$ introduced in (47) and 49

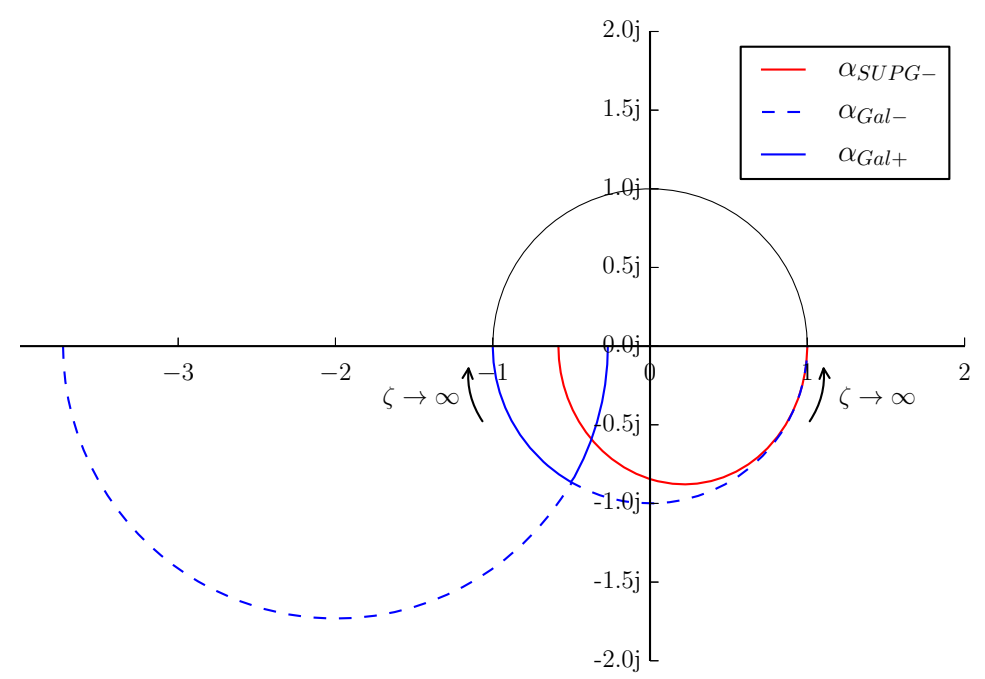

Figure 13: Variation of the coefficients $\alpha$ in the complex plane for $\zeta>0$ 
Now, the property of non-reflectivity of a homogeneous Dirichlet boundary condition when using SUPG stabilization can be shown. Consider a segment of length $L$, discretized by $N$ segments of length $h$, with nodes number ranging from 0 to $N$. On the node $N$, a homogeneous Dirichlet boundary condition on all variables is applied. Thus all characteristic variables are also 0 . On the node 0 , a characteristic boundary condition is applied, and imposes 1 on the incoming characteristic and 0 on the others. To simplify, only the second characteristic, which is the incoming one, is considered. The two boundary conditions on the second characteristic, the exponent 2 notation being dropped once again for clarity reason, are

$$
\left\{\begin{array}{l}
w_{0}=1 \\
w_{N}=0
\end{array}\right.
$$

As the solution of the recurrence relation is of the form $w_{i}=\alpha_{-}^{i} w_{0}^{-}+$ $\alpha_{+}^{i} w_{0}^{+}$, the two boundary conditions enable to find the starting point $w_{0}^{+}$ and $w_{0}^{-}$of both geometric solutions:

$$
\left\{\begin{array}{l}
w_{0}^{-}+w_{0}^{+}=1 \\
\alpha_{-}^{N} w_{0}^{-}+\alpha_{+}^{N} w_{0}^{+}=0
\end{array}\right.
$$

If $\alpha_{+}^{N}-\alpha_{-}^{N} \neq 0$, its solutions are

$$
\left\{\begin{array}{l}
w_{0}^{+}=\frac{\alpha_{-}^{N}}{\alpha_{-}^{N}-\alpha_{+}^{N}} \\
w_{0}^{-}=\frac{\alpha_{+}^{N}}{\alpha_{+}^{N}-\alpha_{-}^{N}}
\end{array}\right.
$$

For the equations stabilized with SUPG, the large modulus difference between $\alpha_{+}$and $\alpha_{-}$as indicated by their limit shows that $\alpha_{+}^{N}-\alpha_{-}^{N} \neq 0$. It also demonstrates that $\left|w_{0}^{+}\right| \ll 1$ and $w_{0}^{-} \sim 1$ for large $N$ and $\zeta$. As a reminder, the solution is $w_{i}=\alpha_{+}^{i} w_{0}^{+}+\alpha_{-}^{i} w_{0}^{-}$. Thus, the SUPG solution is carried only by the well-behaved $\alpha_{S U P G-}$, and the divergent component is here only to cancel the solution at the end of the segment. This solution is non-oscillatory, as the real part of both $\alpha$ is positive. It is interesting to note that the SUPG scheme is unstable, as $\left|\alpha_{S U P G+}\right|>1$, but the boundary conditions select only the stable root for the recurrence relation, and so in practice, the scheme is stable.

For the standard Galerkin discretization, $\alpha_{+}$and $\alpha_{-}$converge respectively to -1 and 1 . An analysis using equivalents shows that $\alpha_{+}^{N}-\alpha_{-}^{N}=(-1)^{N}-$ $1+j \frac{N}{\zeta}\left(\frac{(-1)^{N}}{3}-1\right)+o\left(\frac{1}{\zeta^{2}}\right)$. This proves that it is never zero, and that the solution is highly dependent on the parity of $N$. In all cases, the solution is more or less evenly distributed on a oscillatory component $\left(\alpha_{\text {Gal+ }}\right)$ and a sine wave one $\left(\alpha_{\text {Gal- }}\right)$. This makes this scheme unusable, as the amplitude of the smooth component is not the real amplitude of the pressure wave and that the noise is of the same order of magnitude as the real signal. 
The diffusion and the dispersion of the SUPG scheme can be studied through $\alpha$. The diffusion of the scheme relates to the norm of $\alpha$ and the dispersion to its argument. Using the Landau notation, for $\zeta \rightarrow \infty$,

$$
\left|\alpha_{S U P G}\right|=1-\frac{1}{24 \zeta^{4}}+o\left(\frac{1}{\zeta^{5}}\right) \quad \arg \left(\alpha_{S U P G}\right)=-\frac{1}{\zeta}-\frac{11}{720 \zeta^{5}}+o\left(\frac{1}{\zeta^{6}}\right)
$$

It is logical that the first equivalent term of $\arg \left(\alpha_{S U P G}\right)$ should be $-1 / \zeta$. After $\lambda / h$ nodes, the argument of $w_{i}$ should be decreased by $2 \pi$. The minus sign comes from the phase relation $\omega t-k x$. The SUPG scheme is slightly diffusive and dispersive. It is interesting to note that there is no term in $1 / \zeta^{2}$ for the modulus of $\alpha_{S U P G}$, nor a term in $1 / \zeta^{3}$ for the argument, which would have been expected. This shows the high accuracy of the SUPG scheme.

\section{Industrial test case}

This aeroacoustic code was tested on an industrial test case taken from the Clean Sky European project [1]. The Clean Sky project is aimed at developing environmentally friendly airplanes, which would burn less fuel and also emit less noise. As part of the Smart Fixed Wing Aircraft, one of the six platforms of the Clean Sky project, Dassault Aviation was working on the integration of aft-body concept for engine noise shielding [13. An innovative U-tail aircraft model equipped with turbine powered simulators was wind-tunnel tested to assess the acoustic shielding effect of the U-tail on a typical business jet with side-mounted engines. The test case was the turbine-powered simulator only.

\subsection{General description}

The geometry of the test case is the one of a turbine-powered simulator. It is a jet engine scaled down to a sixth of the original size, so as to fit on a aircraft model for wind-tunnel test. Some parts are not exactly to scale compared to a real jet engine. The annular duct is thinner in the turbine simulator. The pylon is also bigger, as the piping bringing compressed air to the turbine-powered simulator could not be made smaller. A picture of the engine is presented on figure 14. For reference purpose, the diameter of the exhaust is $20 \mathrm{~cm}$.

To minimize the inaccuracies in the results due to the mesh, the volume mesh was deliberately made very fine. It is designed for propagation of waves at up to $9.6 \mathrm{kHz}$, though no computations were done at this frequency. As the annulus duct is very thin, complex radial mode with a high radial frequency dictated a high mesh refinement on the modal plane and thus inside the turbine. Outside the exhaust, element size slowly increases to the propagation size. Near the far field boundary, element size was increased 


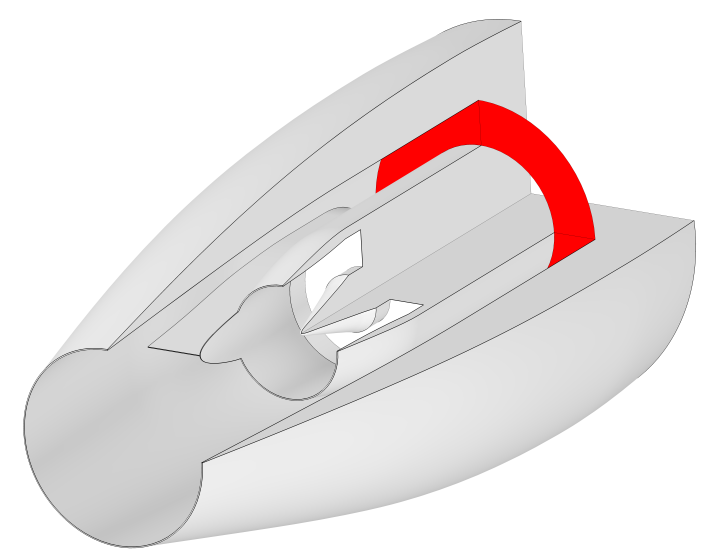

Figure 14: Geometry of the engine with a cut to show the inside. Modal injection is performed on the red plane

slightly to less than ten points per wavelength so as to minimize unwanted reflections.

\subsection{Results without mean flow}

The first calculations were done without mean flow. This case is of little industrial interest, but for being able to compute the solution with another numerical method. When there is no mean flow, acoustic propagation follows the Helmholtz equations. These equations can be solved for instance with a boundary element method (BEM) [16]. An in-house code solving the Helmholtz equations using the BEM method was thus used to give a comparison on this test case. This integral method solves the Helmholtz equations on the surface of the object and does not need a discretization of the volume of the computational domain. It is significantly faster than a finite element method, but it cannot deal with complex mean flows [2].

\subsubsection{Plane wave mode at $2 \mathrm{kHz}$}

A plane wave mode at $2 \mathrm{kHz}$ was first computed. This mode is the least complex one, but still allows to evaluate the performance of the code Aether. The total pressure on the modal plane was imposed to be a plane wave, as described in section 3.1.1. The far-field directivity is pictured on figure 15 . The angle is relative to the exhaust's axis. An angle of $0^{\circ}$ indicates that the point is downstream of the engine in the direction of the jet, while an angle of $180^{\circ}$ means that the point is in front of the engine. There is an excellent agreement between the two methods. For the front lobe, they do not differ by more than a decibel. For angles away from the axis by more than $120^{\circ}$, 

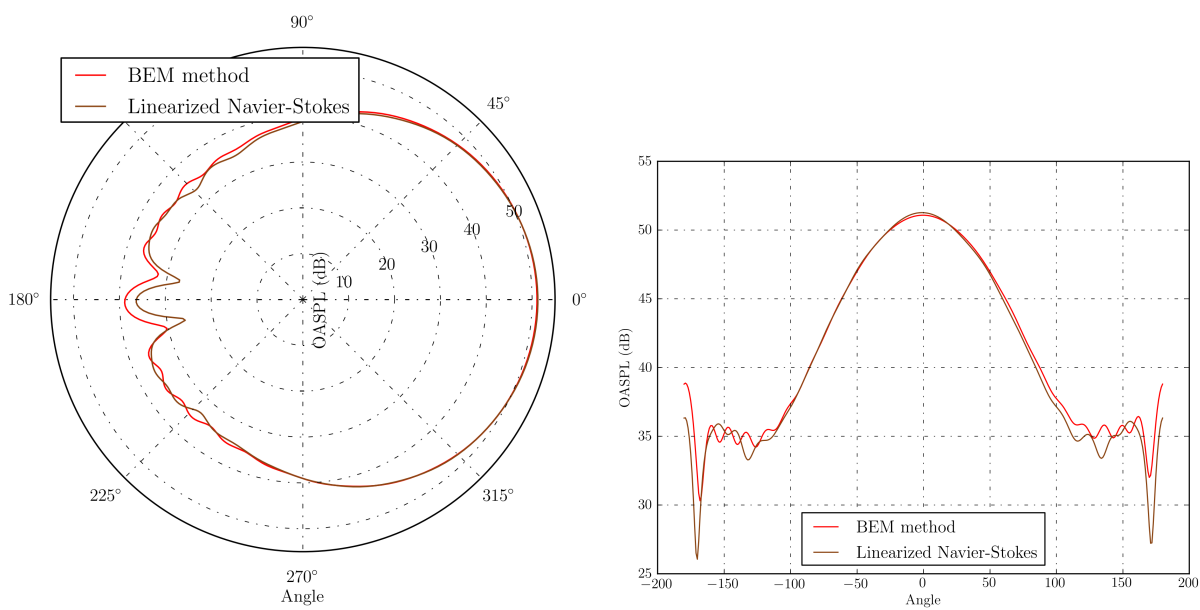

Figure 15: Far-field directivity of a plane mode at $2 \mathrm{kHz}$ without mean flow. Comparison between linearized Navier-Stokes and boundary element method.

diffraction error by the Kirchhoff interpolating surface which is open on the upstream face is significant and explains most of the error between the two curves.

\subsubsection{Complex modes at $2 \mathrm{kHz}$ and $5 \mathrm{kHz}$}

As explained in section 3.2 .2 , other modes than the plane wave can exist in cylindrical geometries. They are more challenging to compute, as they have more complex directivities. To begin with, the $(1,1)$ mode at $2 \mathrm{kHz}$ was computed. Its directivity is shown on figure 16. Once again there is an excellent agreement with the BEM method. On the two lobes, the linearized Navier-Stokes computation is within a decibel of the BEM computation. The only significant difference is the capture of the noise cancellation directly on the axis. Several explanation to this loss of precision can be brought forward. It could either be due to a little bit of numerical diffusion in the volume computation, or an insufficiently converged linear system, or finally the effect of the diffraction in the far field propagation introduced by the openings in the Kirchoff surface. Still, the linearized Navier-Stokes computation is capable of resolving more than $20 \mathrm{~dB}$ of difference between the peak of the lobe and the minimum in the axis.

Higher frequencies are more challenging to compute. For the $(1,1)$ mode, going to $5 \mathrm{kHz}$ changes radically the far-field directivity. Figure 17 shows the directivity at such a frequency. It shows that the code Aether starts to drift away from the BEM method. The two main lobes are not perfectly captured, and there is a distinctive lack of signal for the secondary lobe around $-70^{\circ}$. The main features of the directivity are still captured. Figure 18 shows the 

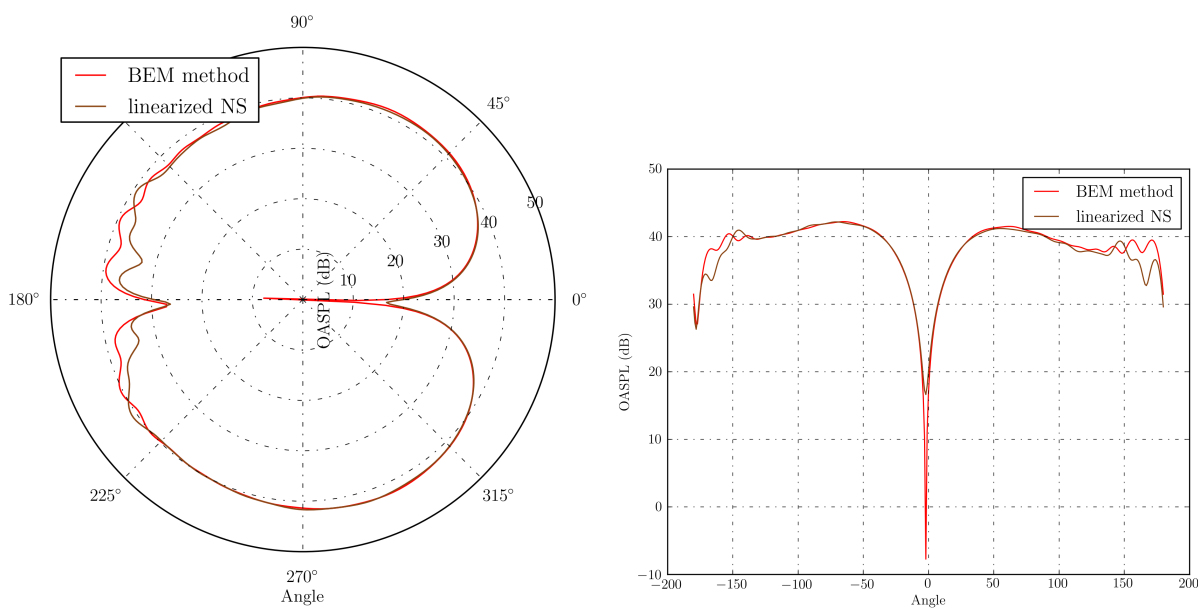

Figure 16: Far-field directivity of a $(1,1)$ mode at $2 \mathrm{kHz}$ without mean flow. Comparison between linearized Navier-Stokes and boundary element method.

real part of the pressure variation in the volume for this mode. It gives an idea of the complexity of the propagation inside the nozzle, and of the complicated interference pattern appearing further away from the exhaust.

\subsection{Results with mean flow}

When a mean flow is added, sound propagation pattern changes completely. Refraction of the sound waves by velocity gradient of the mean flow alters the direction of propagation. Sound waves are refracted away from the axis. The mean flow is not potential, as it represents the jet emitted by the engine, and so the acoustic propagation can no longer be computed using a boundary element method [2].

\subsubsection{Description of the mean flow}

The mean flow was computed using a RANS simulation. As explained in section 4.1. the engine is in fact a small scale model called a turbine powered simulator. To propel the engine and create a jet, compressed nitrogen at low temperature is fed into the model turbine. The jet is thus at around $-100^{\circ} \mathrm{C}$. As a consequence, the sound velocity is very low in the jet, and so the jet is locally supersonic. This is pictured in figure 1 .

\subsubsection{Plane wave mode at $2 \mathrm{kHz}$ with mean flow}

Figure 19 shows the real part of the pressure variation for a plane wave mode at $2 \mathrm{kHz}$. As stated just before, the sound refraction directs the sound energy on the sides. The high amplitude oscillations in the jet correspond to 

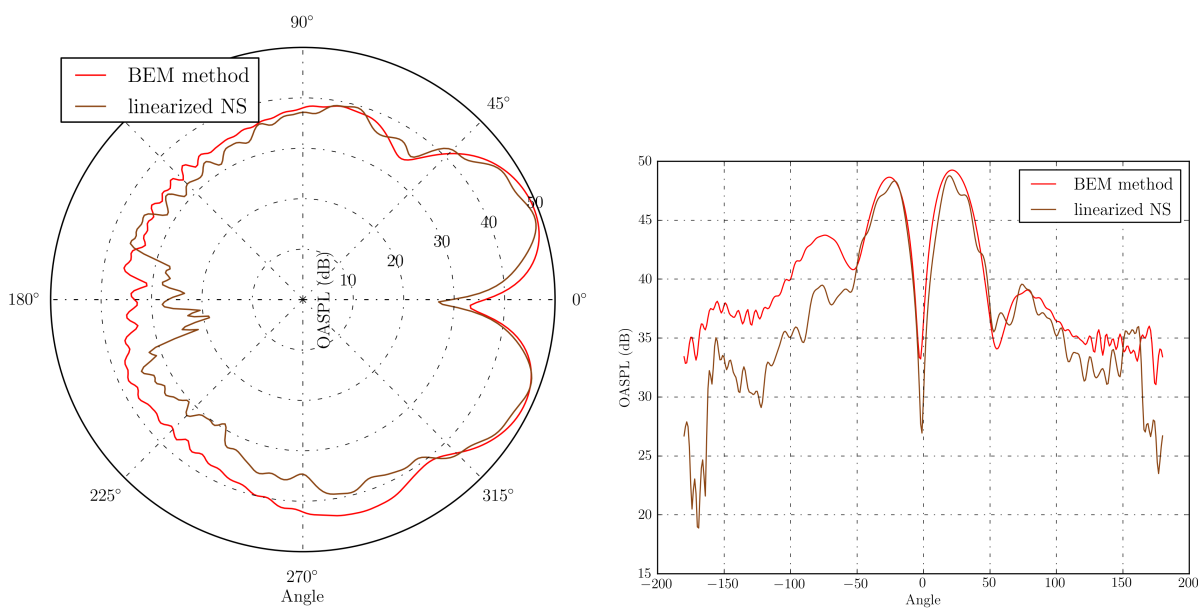

Figure 17: Far-field directivity of a $(1,1)$ mode at $5 \mathrm{kHz}$ without mean flow. Comparison between linearized Navier-Stokes and boundary element method.

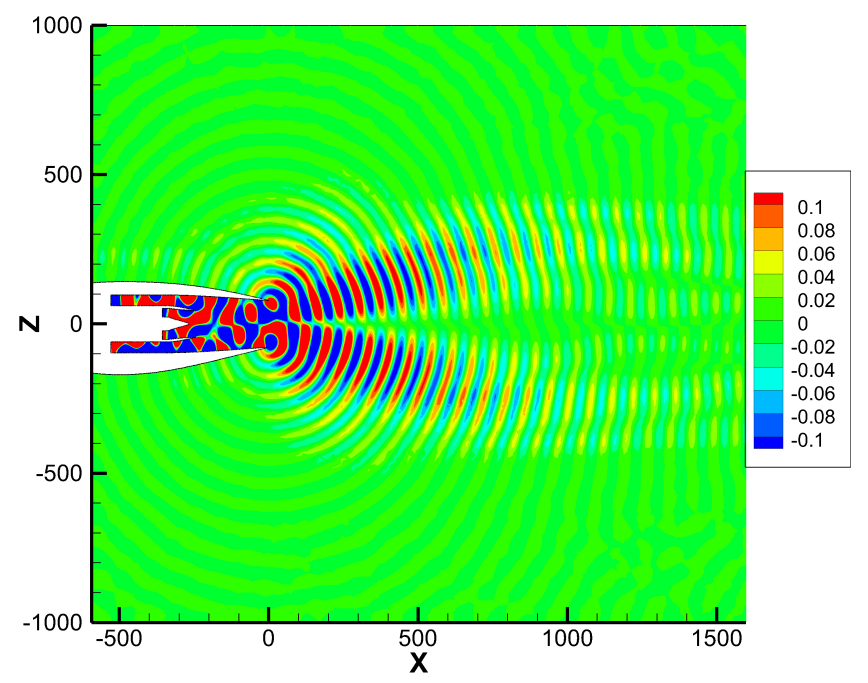

Figure 18: Real part of the pressure variation in Pascal for the mode $(1,1)$ at $5 \mathrm{kHz}$ without mean flow on a slice 


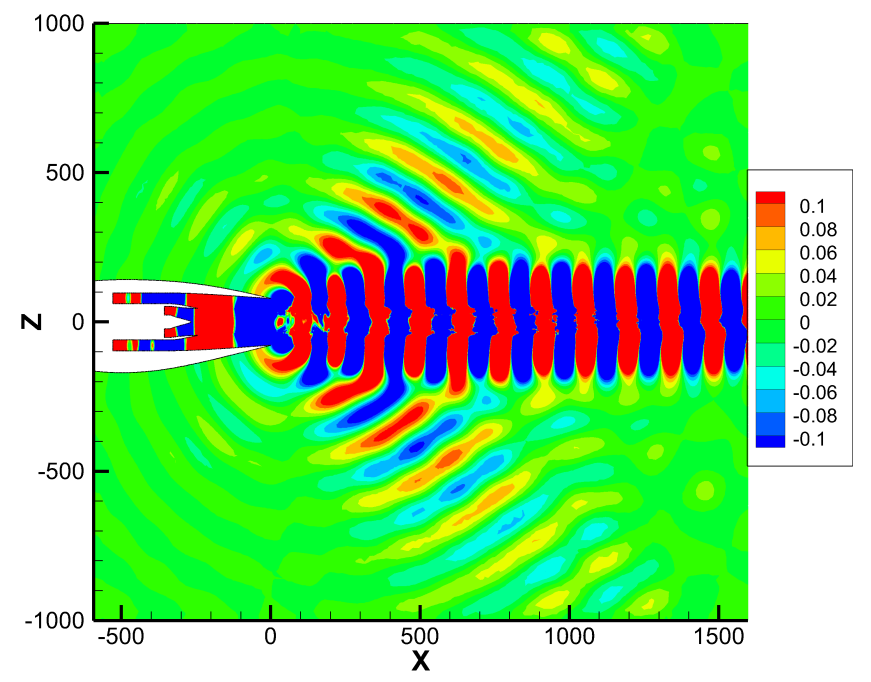

Figure 19: Real part of the pressure variation in Pascal on a slice for a plane wave mode at $2 \mathrm{kHz}$ with the mean flow corresponding to the jet engine at full power

Kelvin-Helmholtz instabilities which are unstable at this frequency. They are located in the shear layer, and they create a high amplitude wave which does not radiate. As the jet expands and is diffused, the shear layers are thicker, and the Kelvin-Helmholtz instability is no longer unstable at this frequency. This means that the amplitude of the wave decreases with the distance to the engine after reaching a maximum. As the transparent boundary conditions are not perfect, care has to be taken that these instability waves are damped sufficiently before they reach the far-field boundary. This proved to be the main driver of the size of the computational domain.

\section{Conclusion}

In this paper, the industrial use of linearized Navier-Stokes equations for propagation of sound in aeroacoustics was demonstrated. A full propagation computation requires first the mean flow solution, then the propagation computation itself using the linearized Navier-Stokes (or linearized Euler) equations, and finally a far-field propagation with the Kirchhoff method. So the full computation uses two volume meshes, one for each of the two first steps, with an interpolation step to transfer the mean flow solution between the two meshes.

The use of entropy variables, which brings better numerical properties, 
introduces some additional complexity when imposing boundary conditions on physical or conserved quantities. The pressure, for instance, depends nonlinearly on the entropy variables. In order to solve the linearized equations, the gradient of these entropy variables is required, which yields a linear relation between the pressure linearized variation, for example, and the linearized variations of the entropy variables. The resulting transformation matrix was used to modify the linear system for imposing a non-homogeneous Dirichlet boundary condition. Its use in a finite element scheme is straightforward. A Dirichlet boundary condition on characteristic variables was implemented in order to impose only the incoming acoustic pressure. A rigorous test using complex modes in a tuned cylinder was devised and showed that the use of normal characteristics is valid even to impose waves with a large angle to the normal.

The SUPG stabilization is the reason why applying homogeneous Dirichlet boundary conditions to all variables leads to a transparent boundary condition. A 1D toy model was used to show that this suprising effect is due to the perfect upwinding on all its characteristics. It also gave a convincing explanation of the good numerical performance of the Aether code in terms of dissipation and dispersion.

Industrial use of linearized Navier-Stokes for propagation of aft fan noise in a jet engine was demonstrated. On a complex geometry, this volume computation led to results which are very close to those obtained by a boundary element method, when no mean flow was present. This assessment demonstrates the numerical efficiency of the Aether code, as far as linearized Navier-Stokes (or linearized Euler) equations are concerned.

\section{Acknowledgment}

This work is supported by a CIFRE PhD fellowship funded in part by ANRT (National Association for Research and Technology) on behalf of the French Ministry of Higher Education and Research, and in part by Dassault Aviation. The authors wish to thank the Clean Sky project, funded by the European Commission, for supporting the work on innovative tailplane for future aircraft.

\section{References}

[1] Clean Sky project. http://www.cleansky.eu.

[2] A. Agarwal and A. P. Dowling. Low-frequency acoustic shielding by the silent aircraft airframe. AIAA Journal, 45(2), February 2007.

[3] F. Chalot. Encyclopaedia of Computational Mechanics, chapter Industrial aerodynamics. Wiley, 2004. 
[4] L. Daumas, Q. Dinh, S. Kleinveld, and G. Rogé. Automatic shape optimization using parametric CAD applied to sonic boom reduction. In 3rd AIAA Multidisciplinary Design Optimization Specialist Conference, pages 23-26, 2007.

[5] L. Daumas, N. Forestier, A. Bissuel, G. Broux, F. Chalot, Z. Johan, and M. Mallet. Industrial frequency-domain linearized Navier-Stokes calculations for aeroelastic problems in the transonic flow regime. In IFASD, 2017.

[6] J. Gikadi, M. Schulze, J. Schwing, S. Föller, and T. Sattelmayer. Linearized Navier-Stokes and Euler equations for the determination of the acoustic scattering behaviour of an area expansion. In 18th AIAA/CAES Aeracoustics Conference. AIAA, 2012.

[7] L. Hascoët and V. Pascual. The Tapenade Automatic Differentiation tool: Principles, Model, and Specification. ACM Transactions On Mathematical Software, 39(3), 2013.

[8] T. J. R. Hughes, L. P. Franca, and M. Mallet. A new finite element formulation for computational fluid dynamics. I. Symmetric forms of the compressible Euler and Navier-Stokes equations and the second law of thermodynamics. Comput. Methods Appl. Mech. Engrg., 54(2):223-234, 1986.

[9] T. J. R. Hughes and M. Mallet. A new finite element formulation for computational fluid dynamics. III. The generalized streamline operator for multidimensional advective-diffusive systems. Comput. Methods Appl. Mech. Engrg., 58(3):305-328, 1986.

[10] M. Mallet. A finite element method for computational fluid dynamics. $\mathrm{PhD}$ thesis, Stanford, 1985.

[11] S. Miller, P. J. Morris, and Y. Zhao. Prediction of fan exhaust noise propagation. In 15th AIAA/CAES Aeracoustics Conference. AIAA, 2009 .

[12] P. Rao and P. J. Morris. Use of finite element methods in frequency domain aeroacoustics. AIAA Journal, 44(7), July 2006.

[13] F. Rey. Design and test of innovative after-bodies for bizjets. Greener aviation, October 2016.

[14] S. W. Rienstra and W. Hirschberg. An Introduction to Acoustics. Eindhoven University of Technology, 2004.

[15] Y. Saad and M. Schultz. GMRES: A generalized minimal residual algorithm for solving nonsymmetric linear systems. SIAM J. Sci. Stat. Comput., 7(3), July 1986. 
[16] Stefan Sauter and Christoph Schwab. Boundary Element Method. Springer Series in Computational Mathematics. Springer, 2011.

[17] Farzin Shakib. Finite element analysis of the compressible Euler and Navier-Stokes equations. PhD thesis, Stanford, 1988.

[18] S. Tsynkov. Numerical solution of problems on unbounded domains. a review. Appl. Num. Math., 27:465 - 532, 1998.

[19] P. Yser, C. Bailly, S. Barré, F. Chalot, and F. Dagrau. High-order Variational Multiscale Model with an Explicit Filtering in a Stabilised Finite Element method for LES/DES Computations. In 22nd AIAA/CEAS Aeroacoustics Conference. AIAA, 2016. 